\title{
II. The Cell Size Distribution and Semigroups of Linear Operators
}

\author{
O. Diekmann
}

In this chapter we present relevant parts of the theory of strongly continuous semigroups of linear operators in the context of a concrete example: the time evolution of the size distribution of a proliferating cell population. Our aim is to give a motivated introduction to the general mathematical theory of linear semigroups and to demonstrate its usefulness for the study of density independent structured population models. We want to show that abstract arguments and concrete calculations may be combined to arrive at strong conclusions.

In section 1 we perform a preliminary transformation of the cell size density equation introduced in section I.4, and we specify the population state space in which we want to work. In section 2 we collect some basic definitions and results from semigroup theory and we illustrate these by means of some elementary but enlightening examples. Subsequently we show in section 3 that one can indeed associate a semigroup with the size structured cell model. Notions from spectral theory are introduced in section 4 and subsequently it is shown that the spectrum of the infinitesimal generator of the cell semigroup can be found by solving a so-called characteristic equation. This characteristic equation is studied in section 5 , and in section 6 we show that the state space can be decomposed according to a subdivision of the spectrum. Sections 7 and 8 are again concerned with the general theory. First we discuss various relations between the spectrum of the generator and the spectrum of the semigroup operators, and then we deal with exponential estimates. In section 9 the results of the preceding sections are applied to the cell model in the special case that $V(2 x)<2 V(x)$, where $V$ is the individual cell growth rate as a function of size $x$. The conclusion is that asymptotically for large time the population grows exponentially, while its size distribution converges towards a (stable) distribution which is independent of the initial condition (the dynamics is asymptotically one-dimensional). Section 10 continues the introduction in section I.3.4 of integration along characteristics, a very important auxiliary technique. The results are used in section 11 to study the exceptional case that $V(2 x)=2 V(x)$ for all relevant $x$. In section 12 the case that $V(2 x)=2 V(x)$ in some interval and $V(2 x)<2 V(x)$ in another interval is treated, and the conclusions for the general case are summarized. Some clarifying remarks about the role of positivity are made in section 13 . Finally, we show in section 14 that the linear theory can be used in a rather special case of a general model for substrate limited growth in a chemostat.

\section{Formulation of the problem.}

In chapter I, section 4, we showed that the size-density of a population of unicellular organisms reproducing by binary fission is governed (under constant environmental conditions) by the linear functional-partial differential equation

$$
\begin{aligned}
& \frac{\partial}{\partial t} n(t, x)=-\frac{\partial}{\partial x}(V(x) n(t, x))-\mu(x) n(t, x)-b(x) n(t, x)+4 b(2 x) n(t, 2 x) \\
& n\left(t, \frac{1}{2} a\right)=0
\end{aligned}
$$

where $V, \mu$ and $b$ denote, respectively, the growth, death and fission rate of individual cells and $a=\min$ support of $b$ = minimal size at which fission can possibly occur. In order to describe that cells will divide with certainty before reaching a maximal size, which we take without loss of generality to be $x=1$, we shall assume that $b$ has a nonintegrable singularity in $x=1$ and we shall restrict the domain of the size variable $x$ to the interval $[1 / 2 a, 1]$. We repeat convention I.4.2.1 as:

CONVENTION 1.1. If the $x$-argument of a function exceeds one, the value of the function is by definition zero.

This convention serves to assign to the term $4 b(2 x) n(t, 2 x)$ the value zero for $x>\frac{1}{2}$, in accordance with the fact that no baby cell will have size greater than one half. Throughout this chapter we assume that $0<a<1$, but in many 
parts we make the much stronger assumption $\frac{1}{2} \leqslant a<1$ which entails that the smallest mother is still larger than the biggest daughter.

For each fixed $t$ we conceive of $n(t, \cdot)$ as a function defined on $\left[\frac{1}{2} a, 1\right]$.

INTERLUDE 1.2. About Banach spaces and linear operators: a quick introduction for readers with little mathematical background. It is convenient to consider such a function of $x$ as an element of (point in) a space in much the same way as an $N$-tuple of real numbers is considered as a point in $\mathbb{R}^{N}$, the $N$-dimensional Euclidean space. A space of functions on $\left[\frac{1}{2} a, 1\right]$ will necessarily be infinite-dimensional. Quite naturally we want it to be a linear space: if we multiply an element by a scalar (a real or complex number) we want to obtain another element and likewise if we add two elements.

It turns out that in an infinite dimensional space one can introduce several non-equivalent notions of convergence. So we have to specify explicitly our ideas about nearness. A convenient way to do this is to define the distance between two elements, in such a way that certain natural (intuitive) geometrical properties hold. In a normed linear space $X$ there is associated with each element $f$ of $X$ (notation: $f \in X$ ) a nonnegative real number $\|f\|$, called the norm of $f$, such that

(i) $\|f+g\| \leqslant\|f\|+\|g\|$,

(ii) $\|\alpha f\|=|\alpha|\|f\|$ for any scalar $\alpha$,

(iii) $\|f\|=0$ implies $f=0$.

In such a space we can identify the distance between $f$ and $g$ with $\|f-g\|$. Note that by (i) the triangle inequality

$$
\|f-g\| \leqslant\|f-h\|+\|h-g\|
$$

holds. Finally we want to include in our axioms the technical condition that sequences for which it is reasonable to expect that they converge, do indeed converge to an element of the space. An infinite sequence $\left\{f_{n}\right\}$ of elements of $X$ is called a Cauchy sequence if for any $\epsilon>0$ there exists an integer $n_{0}$ such that the relations $k \geqslant n_{0}$ and $l \geqslant n_{0}$ imply that $\left\|f_{k}-f_{l}\right\|<\epsilon$. A Banach space $X$ is a normed linear space in which every Cauchy sequence $\left\{f_{n}\right\}$ converges to some $f \in X$ (i.e. $\left\|f_{n}-f\right\| \rightarrow 0$ for $n \rightarrow \infty$ ).

The defining properties of a Banach space are such that:

(i) they allow the construction of a rich and powerful mathematical theory,

(ii) many concrete function spaces, which arise in practical applications, have these properties.

Function spaces which we shall meet in the following are:

(i) $C[\alpha, \beta]$, the space of continuous functions defined on the interval $[\alpha, \beta]$ with values in $\mathbb{R}$ provided with the supremum norm $\|f\|=\sup \{|f(x)| \mid \alpha \leqslant x \leqslant \beta\}$

(ii) $\quad C_{0}[\alpha, \beta]$, the subspace of $C[\alpha, \beta]$ of functions which are zero for $x=\alpha$, provided with the supremum norm;

(iii) $L_{p}(\alpha, \beta)$, the space of integrable functions for which $\|f\|=\left(\int_{\alpha}^{\beta}|f(x)|^{p} d x\right)^{\frac{1}{p}}$ is finite (strictly speaking this is a space of equivalence classes of functions; see RuDIN, 1974);

(iv) $L_{\infty}(\alpha, \beta)$, the space of (essentially) bounded measurable functions provided with the supremum norm.

Let $X$ and $Y$ be Banach spaces with norms \|\|$_{X}$ and \|\|$_{Y}$ and let $L$ be a linear operator from $X$ into $Y$ (that is, to every $f \in X$ there is associated $L f \in Y$ and $L(\alpha f)=\alpha L f$ and $L(f+g)=L f+L g$ ). To say that $L$ is continuous means that $L f_{n}$ converges to $L f$ in $Y$, whenever $f_{n}$ converges to $f$ in $X$, but one can prove that this property is equivalent to $L$ being bounded in the sense that for some positive constant $K$

$$
\|L f\|_{Y} \leqslant K\|f\|_{X} \text { for all } f \in X .
$$

Hence we use for linear operators the words "continuous" and "bounded" interchangeably. The set of all bounded linear operators from $X$ into $Y$ is itself a linear space $\mathscr{Q}(X, Y)$ which we can norm by

$$
\|L\|_{L(X, Y)}:=\sup \left\{\|L f\|_{Y} \mid\|f\|_{X} \leqslant 1\right\} .
$$

One can prove that equipped with this so-called operator norm $L(X, Y)$ is a Banach space. An alternative equivalent definition of the operator norm is

$$
\|L\|_{L(X, Y)}=\inf \{K \mid \text { relation (1.2) holds }\} .
$$

Frequently we will omit the indices $X, Y$ and $L(X, Y)$, which distinguish the various norms from each other, simply because the context unambiguously stipulates which norm is meant.

It may happen that one can define a linear operator $L$ on a subspace of $X$ without being able to extend $L$ to a bounded operator defined on all of $X$ (we will meet several examples below). In such a case $L$ is said to be unbounded and one has to specify carefully both the subspace on which $L$ is defined, to be denoted by $\mathscr{D}(L)$ and to be called the domain of $L$, and the action of $L$. If the domains of two unbounded operators are not identical they have to be considered as different objects even if their action is 
identical. Frequently $\mathscr{D}(L)$ will be dense in $X$ (i.e. for any $f \in X$ and any $\epsilon>0$ one can find $g \in \mathscr{Q}(L)$ such that $\|f-g\|<\epsilon$ or, equivalently, the closure $\overline{\mathscr{D}(L)}$ of $\mathscr{D}(L)$ is all of $X)$.

Occasionaly we shall have to integrate Banach space valued functions (see, for instance, the variation-of- constants formula (3.6) below). For our purposes the Riemann 'integral of a continuous function is adequate. We refer to LADAS \& LAKSHMIKANTHAM (1972) for a quick introduction to the theory of integration in Banach space.

It remains to specify the function space $Y$ in which $t \mapsto n(t, \cdot)$ is supposed to take its values. At this point many roads depart. From the point of view of probability theory one may wish to interpret $n(t, \cdot)$ as a measure. Our "definition" of $n$ in section 1.4 suggests to take $L_{1}\left(\frac{1}{2} a, 1\right)$ as the state space $Y$. But continuous functions may be easier to work with.

Likewise we have to make assumptions about the measurability, integrability and/or continuity of $V, \mu$ and $b$ and we can either strive for the utmost generality by assuming as little as possible about these ingredients of our model or, on the contrary, we may assume everything which seems reasonable and makes life easy. Noting that it is largely a matter of taste we adopt the second attitude. We make the

\section{ASSUMPTION 1.3:}

$H_{V}: V$ is a strictly positive continuous function on $\left[\frac{1}{2} a, 1\right]$;

$H_{\mu}: \mu$ is a non-negative continuous function on $\left[\frac{1}{2} a, 1\right]$;

$H_{b}: b$ is non-negative and continuous on $\left[\frac{1}{2} a, 1\right) ; b(x)=0$ for $x \in\left[\frac{1}{2} a, a\right]$ and $b(x)>0$ for $x \in(a, 1)$ while $\lim _{x \uparrow 1} \int_{a}^{x} b(\xi) d \xi=+\infty$.

Note that one of the assumptions concerning $b$ expresses that every cell with size greater than $a$ has a positive probability per unit of time of division.

Before we define $Y$ we make a transformation. Motivated by the cohort calculations in subsection I.4.1 we define

$$
E(x)=\exp \left[-\int_{a / 2}^{x} \frac{b(\xi)+\mu(\xi)}{V(\xi)} d \xi\right) .
$$

The transformation

$$
m(t, x)=\frac{V(x)}{E(x)} n(t, x)
$$

leads to

$$
\left\{\begin{array}{l}
\frac{\partial}{\partial t} m(t, x)=-V(x) \frac{\partial}{\partial x} m(t, x)+k(x) m(t, 2 x) \\
m\left(t, \frac{1}{2} a\right)=0
\end{array}\right.
$$

where

$$
k(x)=4 \frac{V(x)}{E(x)} \frac{E(2 x)}{V(2 x)} b(2 x), \frac{1}{2} a \leqslant x \leqslant \frac{1}{2} .
$$

EXERCISE 1.4: Check that (1.7) is correct.

We emphasize that Convention 1.1 implies that $k(x) m(t, 2 x)=0$ for $x>\frac{1}{2}$ and for convenience later on we define

$$
k(x)=0, \quad x>\frac{1}{2} .
$$

The profit of transformation (1.6) is technical in character: it turns out that (1.7) is easier to handle than (1.1). We can interpret $m$ as the, with a survival-factor corrected, flux of cells (recall that the flux at $x$ is by definition the number of cells which pass $x$ per unit of time and therefore equals $V(x) n(t, x)$, the velocity times the density).

We are going to look for a "solution" $\left.t \rightarrow m(t,)^{\cdot}\right)$ of $(1.7)$ which takes values in the Banach space $X$ of continuous functions defined on $\left[\frac{1}{2} a, 1\right]$ which are zero in $x=\frac{1}{2} a$, provided with the supremum norm: 


$$
X=\left\{\psi \in C\left[\frac{1}{2} a, 1\right] \mid \psi\left(\frac{1}{2} a\right)=0\right\}=C_{0}\left[\frac{1}{2} a, 1\right] .
$$

This amounts to considering $n(t, \cdot)$ as an element of a weighted $C$-space $Y$ with tailor-made norm

$$
\|\phi\|=\sup \left\{\frac{V(x)}{E(x)}|\phi(x)| \mid \frac{1}{2} a \leqslant x \leqslant 1\right\} .
$$

In particular it implies that $n(t, x)$ has to go to zero for $x \uparrow 1$ at least as fast as $E(x)$ and that the behaviour near $x=1$ carries extra weight (note that $E(1)=0$ !). In view of the cohort calculations these are natural properties. Moreover, as an a posteriori justification we shall demonstrate in subsequent sections that these properties are preserved in the course of time (so working in $Y$ is a restriction on the initial condition $n(0, x)$ only). chapter:

We are now ready to give a precise mathematical formulation of the problem and to outline the program of this

(i) we want to interpret (1.7) as an abstract equation

$$
\frac{d m}{d t}=\tilde{A} m
$$

in $X$, where $\tilde{A}$ is the generator of a semigroup $\tilde{T}(t)$ acting on $X$

(ii) we want to study the large time behaviour of $\tilde{T}(t)$

(iii) as an important tool in (ii) we shall use a detailed study of the spectral properties of $\dot{A}$.

REMARK 1.5. The tildes serve here to emphasize the distinction with the generator $\mathrm{A}$ and the semigroup $T(t)$ corresponding to $n(t, \cdot)$ and introduced in section I.5. In the following sections we will omit the tildes.

EXERCISE 1.6. Define $H: Y \rightarrow X$ by (cf. (1.6))

$$
(H \phi)(x)=\frac{V(x)}{E(x)} \phi(x) .
$$

Convince yourself that $H$ is continuous and that its inverse $H^{-1}$ is continuous as well ( $H$ is an isomorphism). Verify that

$$
T(t)=H^{-1} \tilde{T}(t) H
$$

REMARK 1.7. (About the notation.) In the following we shall use the symbols $\lambda, \phi, \psi$ and $k$ to denote mathematical objects which are different from the various distributions and the population growth rate which were denoted by these symbols in section I.4. We trust that this will not lead to confusion.

\section{Strongly continuous semigroups of bounded linear operators}

In the first part of this section and in sections 7 and 8 we present without proof some basic mathematical material concerning linear semigroups of operators. For proofs and additional results we refer to PAZY (1983a) and DAVIES (1980). The bible remains Hille \& Phillips (1957). Other convenient sources are BalaKrishnan (1976), Bell.ENIMorante (1979), Butzer \& Berens (1967), Dunford \& Schwartz (1958), Kato (1976), Ladas \& Lakshmikantham (1972), SchappaCher (1983), WebB (1985a) and Yosida (1980). The second part of this section is devoted to some examples (in particular translation semigroups) which serve to pave the way for our treatment of the fission equation in section 3 .

Let $X$ be a Banach space with norm $\|\cdot\|$. Let $\{T(t)\}_{t \geqslant 0}$ be a family of bounded linear operators from $X$ into itself. We will call $\{T(t)\}$ a strongly continuous (one-parameter) semigroup iff

(i) $T(0)=I$

(ii) $T(t) T(\tau)=T(t+\tau), t, \tau \geqslant 0$,

(iii) $\lim _{t \downarrow 0}\|T(t) \phi-\phi\|=0, \forall \phi \in X$.

(The adverb "strongly" indicates that (iii) amounts to convergence in the strong operator topology). Assumption (iii) is based on the wish to be assured of continuous orbits $t \mapsto T(t) \phi$ and so it may seem strange that (iii) only requires continuity in $t=0$. Therefore we propose

EXERCISE 2.1. Show that (i) - (iii) guarantee that for each fixed $\phi \in X$ the mapping $t \mapsto T(t) \phi$ is continuous from $\mathbb{R}_{+}$to $X$. 
The operator $A$ defined by

$$
A \phi=\lim _{t \downarrow 0} \frac{1}{t}(T(t) \phi-\phi)
$$

with domain of definition $\mathscr{Q}(A)$ consisting of those $\phi \in X$ for which $\frac{1}{t}(T(t) \phi-\phi)$ converges in $X$ to a limit as $t \downarrow 0$, is called the infinitesimal generator of $\{T(t)\}$. We will frequently omit the adjective "infinitesimal".

THEOREM 2.2. (Uniqueness of the correspondence). A linear operator can be the generator of at most one semigroup.

Definition 2.3. A linear operator $L: X \rightarrow Y$ (where $X$ and $Y$ are Banach spaces) is called closed iff for each sequence $\left\{x_{n}\right\}$ in $X$ such that (i) $x_{n} \in \mathcal{D}(L)$; (ii) $\exists x \in X$ such that $x_{n} \rightarrow x$ for $n \rightarrow \infty$; (iii) $\exists y \in Y$ such that $L x_{n} \rightarrow y$; necessarily $x \in \mathscr{Q}(L)$ and $L x=y$.

REMARXs (i) One can show that $L$ is closed iff the graph $\{(x, L x) \mid x \in \mathscr{D}(L)\}$ is a closed subset of $X \times Y$.

(ii) Among unbounded operators some behave better than others. We will see later that closed operators are good guys in the sense that for these a satisfactory spectral theory exists.

(iii) Differential operators are, as a rule, closed.

In principle the generator $A$ of a semigroup $\{T(t)\}$ could be a "bad" operator with a rather small domain of definition. The next result states that this is actually impossible.

THEOREM 2.4. $A$ is a closed operator and $\mathcal{D}(A)$ is dense in $X$.

Intuitively $A$ is the derivative of $T(t)$ at $t=0$. Indeed we have

THEOREM 2.5. If $\phi \in \mathscr{D}(A)$ then $T(t) \phi \in \mathscr{Q}(A)$ for all $t \geqslant 0$. On $\mathscr{D}(A)$ the operators $T(t)$ and $A$ commute and

$$
\frac{d}{d t} T(t) \phi=A T(t) \phi=T(t) A \phi, \text { if } \phi \in D(A) .
$$

One of the high-lights of semigroup theory gives a precise characterization of those operators which generate semigroups:

THEOREM 2.6. (Hille-Yosida). A closed operator $A$ with dense domain $\mathscr{D}(A)$ is the infinitesimal generator of a strongly continuous semigroup iff real numbers $M$ and $\omega$ exist such that for all $\lambda>\omega$ the operator $\lambda I-A$ has $a$ bounded inverse and

$$
\left\|\left((\lambda I-A)^{-1}\right)^{n}\right\| \leqslant \frac{M}{(\lambda-\omega)^{n}}, n=1,2, \cdots
$$

REMARKS (i) Of course the symbol $\|\cdot\|$ here indicates the operator norm (see Interlude 1.2).

(ii) The most important way to establish that some given operator generates a semigroup is to verify the Hille-Yosida conditions (see PAZY, 1983a). However, for the kind of problems we are dealing with in this chapter there exist, as we will show in detail, easier ways. As a consequence we are not going to use Theorem 2.6 (it is stated here only for the sake of completeness).

EXAMPLE 2.7. Let $X=B U C(\mathbb{R})$, the space of bounded, uniformly continuous functions from $\mathbb{R}$ into $\mathbb{R}$ provided with the supremum norm $\|\phi\|=\sup \{|\phi(x)| \mid-\infty<x<+\infty\}$. Defining

$$
(T(t) \phi)(x)=\phi(x-t)
$$

we clearly obtain a strongly continuous semigroup (note that the strong continuity of translation is guaranteed by our. restriction to uniformly continuous functions).

Claim: $A \phi=-\phi^{\prime}$, with $\mathscr{D}(A)=\left\{\phi \mid \phi\right.$ is continuously differentiable and $\phi^{\prime}$ belongs to $\left.B U C(\mathbb{R})\right\}$.

Proof. Suppose that $\phi \in \mathscr{Q}(A)$ and $A \phi=\psi$ then by definition

$$
\lim _{t \downarrow 0} \sup \left\{\left|\frac{\phi(x-t)-\phi(x)}{t}-\psi(x)\right| \mid-\infty<x<+\infty\right\}=0,
$$

from which we conclude that $\psi$ is the left-derivative of $-\phi$. Since $\psi$ is (uniformly) continuous the inequality 


$$
\left|\frac{\phi(x+t)-\phi(x)}{-t}-\psi(x)\right| \leqslant\left|\frac{\phi(y-t)-\phi(y)}{t}-\psi(y)\right|+|\psi(y)-\psi(y-t)|,
$$

where $y=x+t$, shows that $\phi$ is differentiable with derivative $-\psi$. If, on the other hand, $\phi^{\prime} \in B \cup C(\mathbb{R})$ then

$$
\left|\frac{\phi(x-t)-\phi(x)}{t}+\phi^{\prime}(x)\right|=1 \int_{0}^{t}\left(\phi^{\prime}(x)-\phi^{\prime}(x-\tau)\right) d \tau \mid \stackrel{t \downarrow 0}{\downarrow 00}
$$

uniformly for $x \in \mathbb{R}$ since

$$
\forall \epsilon>0 \exists \delta>0 \text { such that }\left|\phi^{\prime}(x)-\phi^{\prime}(x-\tau)\right| \leqslant \epsilon \text { provided }|\tau| \leqslant \delta \square
$$

REMARKs (i) $A$ is unbounded. Indeed, consider $\phi(x)=\sin m x$ then $\|\phi\|=1$ and $\|A \phi\|=m$ and consequently for no $K \in \mathbb{R}$ the inequality $\|A \phi\| \leqslant K\|\phi\|$ can hold for all $\phi \in X$.

(ii) The fact that $\mathscr{Q}(A)$ is dense in $X$ is well-known in this special example.

(iii)One can easily verify directly that $A$ is closed by taking limits in the relation $\phi_{n}(x)=\phi_{n}(0)+\int_{0}^{x} \phi_{n}^{\prime}(\xi) d \xi$.

(iv)If $\phi$ is differentiable then so is its translate and the order of translation and differentiation may be changed without changing the result. Moreover, $\frac{d}{d t} \phi(x-t)=-\phi^{\prime}(x-t)$. Compare this with Theorem 2.5 .

(v) Defining $n(t, x)=(T(t) \phi)(x)$ we may, if $\phi \in \mathscr{D}(A)$, rewrite the abstract equation $\frac{d}{d t} T(t) \phi=A T(t) \phi$ as $\frac{\partial n}{\partial t}+\frac{\partial n}{\partial x}=0$, a first order partial differential equation with initial condition $n(0, x)=\phi(x)$. We observe that both partial derivatives exist iff $\phi \in \mathscr{Q}(A)$, but that the semigroup yields a very natural extension of the solution concept. This can be made more explicit by interpreting $\frac{\partial}{\partial t}+\frac{\partial}{\partial x}$ as the directional derivative $D$ in the (1,1) - direction:

$$
D f(t, x):=\lim _{\epsilon \rightarrow 0} \frac{1}{\epsilon}\{f(t+\epsilon, x+\epsilon)-f(t, x)\}
$$

(see Appendix IIIA for a general definition). Indeed, the directional derivative $D n$ exists, even though $n$ is not $C^{1}$, and $D n=0$. Thus $n(t, x)=(T(t) \phi)(x)$ is a solution of $D n=0, n(0, x)=\phi(x)$.

The example above illustrates a general principle: the generator of translation is differentiation. A technical elaboration of this principle in various function spaces amounts to a precise description of the domain of definition of the operator of differentiation. For the next result we refer to RudiN, 1974, Chapter 8.

Definirion 2.8. A function $\phi:[a, b] \rightarrow \mathbb{R}$ is called absolutely continuous if $\forall \epsilon>0 \exists \delta(\epsilon)>0$ such that for each finite collection of disjunct open intervals $\left(x_{1}, y_{1}\right), \cdots,\left(x_{N}, y_{N}\right)$ the condition $\sum_{i=1}^{N}\left(y_{i}-x_{i}\right)<\delta$ implies $\sum_{i=1}^{N}\left|\phi\left(y_{i}\right)-\phi\left(x_{i}\right)\right|<\epsilon$.

THEOREM 2.9. (i) Suppose $\phi(x)=\phi(a)+\int_{a}^{x} \psi(\xi) d \xi$ with $\psi \in L_{1}[a, b]$. Then $\phi$ is absolutely continuous.

(ii) Let $\phi$ be absolutely continuous. Then $\phi$ is differentiable for almost all $x$ and $\phi^{\prime} \in L_{1}[a, b]$ while $\phi(x)=\phi(a)+\int_{a}^{x} \phi^{\prime}(\xi) d \xi$.

EXAMPLE 2.10. Let $X=L_{1}(\mathbb{R})$ and define

$$
(T(t) \phi)(x)=\phi(x-t) .
$$

Then $\{T(t)\}$ is a strongly continuous semigroup (more general the translation operator is continuous in $L_{p}$-spaces with $1 \leqslant p<\infty$; note however that translation is not continuous in $L_{\infty}$ ). In view of the theorem above it comes as no surprise that $A \phi=-\phi^{\prime}$ with $\mathscr{Q}(A)=\left\{\phi \mid \phi\right.$ is absolutely continuous and $\left.\phi^{\prime} \in L_{1}(\mathbb{R})\right\}$.

EXAMPLE 2.11. In bounded domains we have to incorporate a boundary condition. Let $X=L_{1}[0,1]$ and define

$$
(T(t) \phi)(x)= \begin{cases}\phi(x-t) & \text { for } x \geqslant t \\ 0 & \text { for } x<t\end{cases}
$$


Then $A \phi=-\phi^{\prime}$ with $\mathscr{D}(A)=\{\phi \mid \phi$ is absolutely continuous and $\phi(0)=0\}$.

EXAMPLE 2.12. The semigroup of the last example cannot be defined on $C[0,1]$ since, when $\phi(0) \neq 0, T(t) \phi$ would have a jump discontinuity. But with $X=C_{0}[0,1]$, the space of continuous functions on $[0,1]$ which vanish for $x=0$, provided with the supremum norm, everything is fine and $A \phi=-\phi^{\prime}$ with $\mathscr{D}(A)=\left\{\phi \mid \phi \in C^{1}[0,1]\right.$ and $\left.\phi^{\prime}(0)=0\right\}$.

EXERCISE 2.13. Verify the last assertion.

EXAMPLE 2.14. The last two examples above correspond to the first order partial differential equation

$$
\frac{\partial n}{\partial t}+\frac{\partial n}{\partial x}=0
$$

with boundary condition $n(t, 0)=0$ and initial condition $n(0, x)=\phi(x)$. As a next step towards the fission problem we change the equation into

$$
\frac{\partial n}{\partial t}+V(x) \frac{\partial n}{\partial x}=0
$$

where $V$ is a continuous and strictly positive function. In order to show that essentially (in a sense specified below) nothing has changed we try to find a transformation of variables

$$
y=G(x)
$$

which reduces the new problem to the old. Since

$$
\frac{\partial}{\partial y}=\frac{\partial x}{\partial y} \frac{\partial}{\partial x}=\left(G^{-1}\right)^{\prime}(G(x)) \frac{\partial}{\partial x}
$$

we want to choose $G$ such that $\left(G^{-1}\right)^{\prime}(G(x))=V(x)$. Differentiating the identity $G^{-1}(G(x))=x$ we obtain $\left(G^{-1}\right)^{\prime}(G(x)) G^{\prime}(x)=1$ and therefore we choose

$$
G(x)=\int_{a / 2}^{x} \frac{d \xi}{V(\xi)} .
$$

Let $X=C_{0}\left[\frac{a}{2}, 1\right], Y=C_{0}[0, G(1)]$ and define $L: X \rightarrow Y$ by

$$
(L \phi)(y)=\phi\left(G^{-1}(y)\right),
$$

and $L^{-1}: Y \rightarrow X$ by

$$
\left(L^{-1} \psi\right)(x)=\psi(G(x)) .
$$

Let the semigroup $T(t)$ acting on $Y$ be defined by (2.2). Then

$$
\tilde{T}(t)=L^{-1} T(t) L
$$

defines a semigroup on $X$ with generator

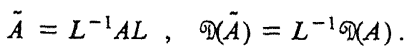

Explicitly we obtain

$$
(\tilde{T}(t) \phi)(x)=\phi\left(G^{-1}(G(x)-t)\right)
$$

(where we define $G^{-1}(y)=0$ for $y \leqslant 0$ ) and

$$
\tilde{A} \phi=-V \phi^{\prime}, \mathscr{D}(\tilde{A})=\left\{\phi \mid \phi \in C^{1}\left[\frac{a}{2}, 1\right] \& \phi\left(\frac{a}{2}\right)=\phi^{\prime}\left(\frac{a}{2}\right)=0\right\} .
$$

EXERCISE 2.15. Verify the preceding calculations. Verify that (2.7) defines a solution of (2.3).

Semigroups $\{\tilde{T}(t)\}$ and $\{T(t)\}$ which are related as in $(2.5)$ are called intertwined or conjugated. In the present example the representation (2.5) shows how the action of $\tilde{T}(t)$ can be decomposed into translation and deformation.

EXERCISE 2.16. If $V$ goes to zero sufficiently fast in the left endpoint of the interval (the "stream in" point) we don't need to prescribe a boundary condition (nor can we). First solve formally

$$
\begin{cases}\frac{\partial n}{\partial t}+x \frac{\partial n}{\partial x}=0 & , 0<x<+\infty \\ n(0, x)=\phi(x) & , 0 \leqslant x<+\infty\end{cases}
$$


and subsequently define the corresponding semigroup on $X=C[0,+\infty)$ and calculate the generator.

EXERCISE 2.17. In continuation of Exercise 1.6 show that

$$
A=H^{-1} \tilde{A} H \text { with } \mathscr{D}(A)=H^{-1} \mathscr{Q}(\tilde{A}) \text {. }
$$

REMARK 2.18. The examples 2.11 and 2.12 show that in an $L_{1}$-space (and more generally in $L_{p}$-spaces) a boundary condition may show up only in the domain of the generator, whereas in the $C$-context one is forced to include it in the definition of the space. But then one of the conditions characterizing the domain of the generator will be a boundary condition for the derivative!

EXERCISE 2.19. Let $A$ be a bounded linear operator from $X$ into $X$. Define for each $t \in \mathbb{R}$ the bounded linear operator $e^{A t}$ by the Taylor series

$$
e^{A t}=\sum_{l=0}^{\infty} \frac{t^{l} A^{l}}{l !},
$$

which converges in the operator norm. Convince yourself that $\left\{e^{A t}\right\}$ is a semigroup with generator $A$.

EXERCISE 2.20. If $X=\mathbb{R}^{N}$ then any bounded linear operator $A$ is, for a given basis, represented by a matrix. One way to compute the matrix $e^{A t}$ is to bring $A$ in Jordan canonical form (HIRSCH \& SMale, 1974) and to use

LEMMA. If $B=P A P^{-1}$ then $e^{B}=P e^{A} P^{-1}$, and

Lemma. If $A_{1} A_{2}=A_{2} A_{1}$ (i.e. $A_{1}$ and $A_{2}$ commute) then $e^{A_{1}} e^{A_{2}}=e^{A_{1}+A_{2}}$.

Show in this manner that

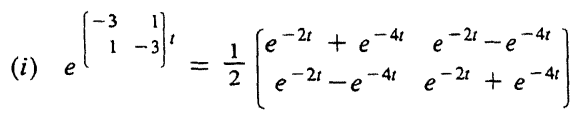

$$
\begin{aligned}
& \text { (ii) } e^{\left(\begin{array}{rr}
\alpha & -\beta \\
\beta & \alpha
\end{array}\right] t}=e^{\alpha t}\left[\begin{array}{cc}
\cos \beta t & -\sin \beta t \\
\sin \beta t & \cos \beta t
\end{array}\right] \text {. }
\end{aligned}
$$

\section{Do growth, death and division generate a semigroup?}

Our aim is to associate a semigroup with the problem

$$
\left\{\begin{array}{l}
\frac{\partial}{\partial t} m(t, x)=-V(x) \frac{\partial}{\partial x} m(t, x)+k(x) m(t, 2 x) \\
m\left(t, \frac{1}{2} a\right)=0 .
\end{array}\right.
$$

Instead of verifying the Hille-Yosida conditions we will first study the rather easy problem obtained by dropping the term $k(x) m(t, 2 x)$ and subsequently re-introduce this term as a relatively innocent perturbation. So we define an unbounded operator $B$ on $X$ by

$$
\left\{\begin{array}{l}
(B \phi)(x)=-V(x) \phi^{\prime}(x) \\
\mathscr{D}(B)=\left\{\phi \in X \mid \phi \in C^{1}\left[\frac{1}{2}, 1\right] \& \phi^{\prime}\left(\frac{1}{2} a\right)=0\right\}
\end{array}\right.
$$

As in Example 2.14 it follows that $B$ generates the strongly continuous semigroup

$$
\left(U_{0}(t) \phi\right)(x)=\phi\left(G^{-1}(G(x)-t)\right)
$$

with

$$
G(x)=\int_{a / 2}^{x} \frac{d \xi}{V(\xi)}
$$

EXERCISE 3.1. Let $F(t)$ be the unique solution of $\frac{d F}{d t}=V(F), F(0)=\frac{1}{2} a$. Interpret $F(t)$ as the size of a cell at time 
$t$ given that the cell had size $\frac{1}{2} a$ at time zero. Verify that $F(t)=G^{-1}(t)$, i.e., $G(F(t))=t$ and $F(G(x))=x$.

EXERCISE 3.2. Interpret $G(x)$ as the time which a cell needs to grow from size $\frac{1}{2} a$ to size $x$.

EXERCISE 3.3. Let $X(t, y)$ denote the solution of the initial value problem $\frac{d x}{d t}=V(x), x(0)=y$. Show that $X(t, y)=G^{-1}(G(y)+t)$ and that, consequently, (3.3) can be rewritten as $\left(U_{0}(t) \phi\right)(x)=\phi(X(-t, x))$. Interpret this representation in biological terms.

EXERCISE 3.4. (a technical point) Convince yourself that the strict positivity of $V$ guarantees that the solution of the initial value problem $\frac{d x}{d t}=V(x), x(0)=y$ is unique (without any Lipschitz condition on $V$ ).

Next we state a perturbation result:

THEOREM 3.5. Let $B$ be the generator of a semigroup $U_{0}(t)$ and let $C$ be a bounded linear operator. Then $B+C$ generates a semigroup $T(t)$.

We shall sketch some instructive aspects of the proof. The differential equation

$$
\frac{d m}{d t}=B m+C m, m(0)=\phi
$$

and the variation-of-constants formula (see HIRSCH \& SMALE, 1974, section V.5) suggest to look for solutions of the integral equation

$$
m(t)=U_{0}(t) \phi+\int_{0}^{t} U_{0}(t-\tau) C m(\tau) d \tau .
$$

Using a certain straightforward estimate (PAZY, 1983a, section 3.1, proposition 1.2) one can show that the successive approximations

$$
m_{k}(t ; \phi)=\sum_{j=0}^{k} U_{j}(t) \phi
$$

where

$$
U_{j+1}(t) \phi=\int_{0}^{t} U_{0}(t-\tau) C U_{j}(\tau) \phi d \tau
$$

converge to a solution $m=m(t ; \phi)$ and, moreover, that there is at most one solution. Finally one can verify that $T(t) \phi=m(t ; \phi)$ defines a strongly continuous semigroup of bounded linear operators with infinitesimal generator $A=B+C, \mathscr{D}(A)=\mathscr{D}(B)$.

Returning to the cell population we formally write

$$
(C \phi)(x)=k(x) \phi(2 x)
$$

and ask ourselves whether or not this defines a bounded operator on $X$.

EXERCISE 3.6. Check that (3.9) defines a bounded operator if and only if $k\left(\frac{1}{2}\right)=0$ or, in other words, $b(x) E(x) \rightarrow 0$ for $x \uparrow 1$. (Hint: recall Convention 1.1).

Although $x b(x) E(x)$ is integrable on $\left[\frac{1}{2} a, 1\right]$, the behaviour of this function in $x=1$ may be such that $C$ is unbounded, and consequently Theorem 3.5 is not strong enough for our purposes. There do exist many generalizations (KATO, 1976). In the present problem one can easily generalize the constructive procedure (3.7) - (3.8) to solve (3.6), by exploiting the embedding of $X$ into $L_{1}\left[\frac{1}{2} a, 1\right]$. Using that (3.3) defines a semigroup on $L_{1}\left[\frac{1}{2} a, 1\right]$ as well, that $C$ is always continuous from $X$ to $L_{1}\left[\frac{1}{2} a, 1\right]$ and that, as one can verify, the integration with respect to $\tau$ produces a continuous function of $x$ one can prove (see DIEKManN, HeIrMans \& THIEME (1984) for the details):

THEOREM 3.7. The operator 


$$
\begin{aligned}
& (A \phi)(x)=-V(x) \phi^{\prime}(x)+k(x) \phi(2 x) \\
& \mathscr{Q}(A)=\left\{\phi \in X \mid \phi \text { is continuously differentiable on }\left[\frac{1}{2} a, \frac{1}{2}\right) \cup\left(\frac{1}{2}, 1\right]\right. \text { and the limits } \\
& \left.\lim _{x \uparrow \frac{1}{2}}-V(x) \phi^{\prime}(x)+k(x) \phi(2 x) \text { and } \lim _{x \downarrow \frac{1}{2}}-V(x) \phi^{\prime}(x) \text { exist and equal each other, and } \phi^{\prime}\left(\frac{1}{2} a\right)=0\right\}
\end{aligned}
$$

is the generator of a semigroup $T(t)$ on $X$.

REMARKS (i) Note that $\mathscr{D}(A)=\mathscr{D}(B)$ if and only if $k\left(\frac{1}{2}\right)=0$ and that, in general, the domains of $B$ and $C$ "interact" to produce $\mathscr{Q}(A)$.

(ii) The terms in the series $T(t) \phi=\sum_{j=0}^{\infty} U_{j}(t) \phi$ have a straightforward biological interpretation. $U_{0}(t) \phi$ is the contribution to the density of those cells which were present at $t=0$ and have not yet divided. We call it the zero"th gen. eration. Inductively we find that $U_{j}(t) \phi$, the $j$-th generation, gives the contribution of those cells which arose from fission of cells of the $(j-1)$ th generation and which have not yet divided themselves. Thus we speak about a generation expansion.

(iii) Note the monotone convergence of the successive approximations (3.7) if $\phi$ is a non-negative function.

(iv) The zero'th generation becomes extinct at $t=G(1)$. One can prove inductively that numbers $e_{j}$ exist such that the $j$ th generation becomes extinct at $t=e_{j}$. Mathematically this amounts to the $j$ th term being identically zero for $t \geqslant e_{j}$.

(v) Assume that $a>\frac{1}{2}$ (i.e., the smallest mother is still larger than the biggest daughter or, in other words, a cell which is just created cannot divide). Since a newly created daughter has a size less than or equal to $\frac{1}{2}$, it needs a certain time to grow up to size $a$ at which it can divide. Consequently there will exist integers $J(\sigma)$ such that for $t \leqslant \sigma$ the generations with $j \geqslant J(\sigma)$ cannot yet exist. Mathematically this amounts to all terms with index $j \geqslant J(\sigma)$ being zero for $t \leqslant \sigma$, i.e., for each finite $t$ the generation expansion has only finitely many non-zero terms. The assumption $a>\frac{1}{2}$ makes the exposition easy but is not needed for the result. One can prove (Diekmann, Heumans \& Thieme, 1984):

LEMMA 3.8. Choose $\sigma>0$. Let the bounded linear operator $H$ from $C([0, \sigma] ; X)$ into itself be defined by

$$
(H m)(t)=\int_{0}^{t} U_{0}(t-\tau) C m(\tau) d \tau
$$

Then $H^{j}=0$ for $j \geqslant \frac{2 \sigma}{a}|V|_{\infty}+m$, where $m$ is such that $2^{-m} \leqslant \frac{a}{2}<2^{-m+1}$ and $|V|_{\infty}=\sup \left\{|V(x)| \mid \frac{1}{2} a \leqslant x \leqslant 1\right\}$. (In other words: $H$ is nilpotent.)

EXERCISE 3.9. (not difficult but time consuming) Prove this lemma for the special case that $a>\frac{1}{2}$ and $g(x)=1$ for all $x$.

In conclusion of this section we address the problem of specifying the sense in which $T(t) \phi$ satisfies the partial differential equation in (3.1). Combining Remark (v) about and below Example 2.7 and the representation (2.5) we now define

$$
(D f)(t, x)=\lim _{\epsilon \rightarrow 0} \frac{1}{\epsilon}\left\{f\left(t+\epsilon, G^{-1}(G(x)+\epsilon)\right)-f(t, x)\right\}
$$

EXERCISE 3.10. Verify that $m(t, x)=(T(t) \phi)(x)$ satisfies

$$
(D m)(t, x)=k(x) m(t, 2 x)
$$

where, to be precise, one should take for $x=\frac{1}{2}$ the limits $\epsilon \uparrow 0$ and $\epsilon \downarrow 0$ in (3.12) separately if $k\left(\frac{1}{2}\right) \neq 0$.

EXERCISE 3.11. Use the identity $G^{-1}(G(x))=x$ and the chain rule to show that $\left(G^{-1}\right)^{\prime}(G(x))=V(x)$.

REMARK. Since $G^{-1}(G(x)+\epsilon)=x+\epsilon V(x)+o(\epsilon)$ we have

$$
(D f)(t, x)=\lim _{\epsilon \rightarrow 0} \frac{1}{\epsilon}\{f(t+\epsilon, x+\epsilon V(x))-f(t, x)\}
$$

if $f$ is smooth. However, the derivative in the direction $(1, V(x))$ at the right hand side may not exist, while still the limit in (3.12) is well defined. In section 10 and Chapter III, section 4 we shall interpret $D$ as a derivative along 


\section{characteristics.}

The first step in our analysis of the fission model is now completed: we showed existence and uniqueness of a solution which depends continuously on the initial data $\phi$. The solution is constructed as a series in which every subsequent term is easily calculated from the preceding one. However, this representation of the solution is not of much help for an analysis of the large time behaviour. We need spectral theory.

EXERCISE 3.12. Apply the constructive proof of Theorem 3.5 to the case where $B$ is the generator of translation in $B U C(\mathbb{R})$ (i.e., the operator called $A$ in Example 2.7) and $(C \phi)(x)=k \phi(x)$. Find an explicit expression for $S(t)$ by evaluating the series.

EXERCISE 3.13. (a little tricky) Repeat the foregoing exercise with $(C \phi)(x)=k(x) \phi(x)$ where $k \in B U C(\mathbb{R})$. Note that the explicit expression for $T(t)$ defines a semigroup for a much wider class of functions $k$.

\section{The spectrum of $A$.}

Let $X$ be a Banach space over the complex numbers $\mathbb{C}$ and let $L$ denote a closed linear operator with domain $\mathscr{D}(L) \subset X$ and range $\Re(L) \subset X$. Let $\lambda$ be a complex number.

DefinITION 4.1. $\lambda$ is an element of the resolvent set $\rho(L)$ iff the resolvent $(\lambda I-L)^{-1}$ exists and is bounded, i.e.,

$$
\begin{aligned}
& \text { (i) } \lambda I-L \text { is one-to-one (injective) } \\
& \text { (ii) } \Re(\lambda I-L) \text { is dense in } X \\
& \text { (iii) }(\lambda I-L)^{-1} \text { is bounded }
\end{aligned}
$$

The so-called spectrum $\sigma(L)$ is by definition the complement of $\rho(L)$. The point spectrum $P \sigma(L)$ is the set of those $\lambda \in \mathbb{C}$ for which $\lambda I-L$ is not one-to-one, i.e., $L \phi=\lambda \phi$ for some $\phi \neq 0$. One then calls $\lambda$ an eigenvalue and $\phi$ an eigenvector corresponding to $\lambda$. The null space $\Im(\lambda I-L)$ is called the eigenspace and its dimension the geometric multiplicity of $\lambda$. The generalized eigenspace $\Re(\lambda I-L)$ is the smallest closed linear subspace that contains $\left.\mathscr{R}(\lambda I-L)^{\gamma}\right)$ for $j=1,2,3, \ldots$ and its dimension is called the algebraic multiplicity of $\lambda$.

Although we will not need the following definitions, we present them for completeness. The continuous spectrum $C \sigma(L)$ is the set of those $\lambda \in \mathbb{C}$ for which $\lambda I-L$ is one-to-one and $\Re(\lambda I-L)$ is dense in $X$ but $(\lambda I-L)^{-1}$ is unbounded. The residual spectrum $R \sigma(L)$ is the set of those $\lambda \in \mathbb{C}$ for which $\lambda I-L$ is one-to-one but $\Re(\lambda I-L)$ is not dense in $X$.

Throughout the rest of this chapter (!) we make

AsSUMPTION 4.2. $a \geqslant \frac{1}{2}$

(see section II.1 for the biological interpretation). It will turn out that the analysis of the spectrum of the operator $A$ defined in (3.10), with $k$ defined in (1.8) and (1.9), is rather simple under this biologically reasonable restriction. Moreover, the results are representative for the general case, which is elaborated in detail in HeIJMANS (1985b).

According to the rules we first try to construct $(\lambda I-A)^{-1}$ for as many $\lambda \in \mathbb{C}$ as possible. Note that we now work in the complexification of $X$ (again denoted by $X$ ) which means that all functions take values in $\mathbb{C}$. The abstract inhomogeneous equation $(\lambda I-A) \psi=f$ implies

$$
\begin{cases}V(x) \psi^{\prime}(x)+\lambda \psi(x)=f(x), & \frac{1}{2} \leqslant x \leqslant 1 \\ V(x) \psi^{\prime}(x)+\lambda \psi(x)=f(x)+k(x) \psi(2 x), & \frac{1}{2} a \leqslant x \leqslant \frac{1}{2} \\ \psi\left(\frac{1}{2} a\right)=0 & \end{cases}
$$

\footnotetext{
- This assumption means that $(\lambda I-L)^{-1}$, which at first is only defined on $\mathscr{R}(\lambda I-L)$, can be extended to a bounded operator defined on the whole space $X$. For closed operators the conditions actually imply that $\Re(\lambda I-L)=X$; so the formulation above is unnecessarily cumbersome in the present context, and was chosen solely because it is the standard formulation.
} 
Using the variation-of-constants formula for ordinary differential equations we can solve the first equation to obtain

$$
\psi(x)=C e^{\lambda\left(G\left(\frac{1}{2}\right)-G(x)\right)}+\int_{\frac{1}{2}}^{x} e^{\lambda(G(\xi)-G(x))} \frac{f(\xi)}{V(\xi)} d \xi
$$

for $\frac{1}{2} \leqslant x \leqslant 1$ with the constant $C$ still to be determined. The right hand side of the second equation in (4.1) can now be expressed in known functions and the unknown constant $C$ and we find, taking account of the third equation,

$$
\psi(x)=\int_{a / 2}^{x} e^{\lambda(G(\xi)-G(x))}\left\{C e^{\lambda\left(G\left(\frac{1}{2}\right)-G(2 \xi)\right)} k(\xi)+f(\xi)+k(\xi) \int_{\frac{1}{2}}^{2 \xi} e^{\lambda(G(\eta)-G(2 \xi))} \frac{f(\eta)}{V(\eta)} d \eta\right\} \frac{d \xi}{V(\xi)}
$$

for $\frac{1}{2} a \leqslant x \leqslant \frac{1}{2}$. The first question is whether or not (4.2) and (4.3) define an element $\psi$ of $X$. The only thing that could possibly be wrong is the continuity in $x=\frac{1}{2}$. Now $\lim _{x \downarrow \frac{1}{2}} \psi(x)=C$ and $\lim _{x \uparrow \frac{1}{2}} \psi(x)=\pi(\lambda) C+\zeta(\lambda, f)$, where by definition

$$
\pi(\lambda)=\int_{\frac{1}{2} a}^{\frac{1}{2}} e^{\lambda(G(\xi)-G(2 \xi))} \frac{k(\xi)}{V(\xi)} d \xi
$$

and

$$
\zeta(\lambda, f)=\int_{\frac{1}{2} a}^{\frac{1}{2}} e^{\lambda\left(G(\xi)-G\left(\frac{1}{2}\right)\right)}\left\{f(\xi)+k(\xi) \int_{\frac{1}{2}}^{2 \xi} e^{\lambda(G(\eta)-G(2 \xi))} \frac{f(\eta)}{V(\eta)} d \eta\right\} \frac{d \xi}{V(\xi)} .
$$

In order that $\psi$ is continuous we have to choose $C$ such that

$$
(1-\pi(\lambda)) C=\zeta(\lambda, f) .
$$

If $\pi(\lambda) \neq 1$ we can, for arbitrary $f \in X$, indeed satisfy this compatibility condition. The explicit expressions (4.2) - (4.3) with

$$
C=(1-\pi(\lambda))^{-1} \zeta(\lambda, f)
$$

define a continuous mapping $f \mapsto \psi$ from $X$ into $X$ and our construction guarantees that $\psi \in \mathscr{D}(A)$ and $(\lambda I-A) \psi=f$. Thus we proved

Theorem 4.3. $\pi(\lambda) \neq 1$ implies $\lambda \in \rho(A)$.

Next, consider those $\lambda$ for which $\pi(\lambda)=1$. If we choose $f(x) \equiv 0$ the function $\psi$ defined by (4.2) - (4.3) is, for arbitrary $C \in \mathbb{C}$, an element of $\mathscr{D}(A)$ such that $(\lambda I-A) \psi=0$. Hence we have

ThEOREM 4.4. $\pi(\lambda)=1$ implies $\lambda \in P \sigma(A)$.

So the spectrum of $A$ is precisely the set $\{\lambda \mid \pi(\lambda)=1\}$ and $A$ has only a point spectrum. By analogy with the well-known situation for ordinary differential equations we will call the equation

$$
\pi(\lambda)=1
$$

the characteristic equation. In subsection I.4.3 we discussed its biological interpretation and in the next section we discuss the position of its roots in the complex plane.

EXERCISE 4.5. The assumption $a \geqslant \frac{1}{2}$ made that we had to consider only two subintervals when constructing the resolvent. Use three subintervals to derive the corresponding characteristic equation for the case $a \geqslant \frac{1}{4}$.

The construction above implies that elements of $भ(\lambda I-A)$ are unique modulo the constant $C$. So the dimension of $\Re(\lambda I-A)$ (i.e., the geometric multiplicity) is at most one (here the restriction $a \geqslant 1 / 2$ is essential). In conclusion of this section we prove

ThEOREM 4.6. Suppose $\pi(\lambda)=1$. The eigenvalue $\lambda$ is simple (i.e. has algebraic multiplicity one) iff $\pi^{\prime}(\lambda) \neq 0$. 
Proof. Any element $\phi$ of $\Re\left((\lambda I-A)^{2}\right)$ that does not belong to $\Re(\lambda I-A)$ necessarily satisfies $(\lambda I-A) \phi=\theta \psi$, for some non-zero constant $\theta$, where $\psi$ is defined by (4.2) - (4.3) with $f(x) \equiv 0$ and, say, $C=1$. Just as before we find the solvability condition $(1-\pi(\lambda)) \phi\left(\frac{1}{2}\right)=\theta \zeta(\lambda, \psi)$. Since $\pi(\lambda)=1$ this condition can be satisfied iff $\zeta(\lambda, \psi)=0$. Now $\zeta(\lambda, \psi)=(1)+(2)$ where

$$
\begin{aligned}
& (1)=\int_{\frac{1}{2} a}^{\frac{1}{2}} e^{\lambda\left(G(\xi)-G\left(\frac{1}{2}\right)\right)} \frac{\psi(\xi)}{V(\xi)} d \xi=\int_{\frac{1}{2} a \frac{1}{2} a}^{\frac{1}{2}} e^{\lambda(G(\eta)-G(2 \eta))} k(\eta) \frac{d \eta}{V(\eta)} \frac{d \xi}{V(\xi)}=\int_{\frac{1}{2} a}^{\frac{1}{2}}\left(G\left(\frac{1}{2}\right)-G(\xi)\right) e^{\lambda(G(\xi)-G(2 \xi))} k(\xi) \frac{d \xi}{V(\xi)} \\
& (2)=\int_{\frac{1}{2} a}^{\frac{1}{2}} e^{\lambda\left(G(\xi)-G\left(\frac{1}{2}\right)\right)} k(\xi) \int_{\frac{1}{2}}^{2 \xi} e^{\lambda(G(\eta)-G(2 \xi))} \frac{\psi(\eta)}{V(\eta)} d \eta \frac{d \xi}{V(\xi)}=\int_{\frac{1}{2} a}^{\frac{1}{2}} e^{\lambda(G(\xi)-G(2 \xi))} k(\xi)\left(G(2 \xi)-G\left(\frac{1}{2}\right)\right) \frac{d \xi}{V(\xi)} .
\end{aligned}
$$

Hence $\zeta(\lambda, \psi)=\int_{\frac{1}{2} a}^{\frac{1}{2}} e^{\lambda(G(\xi)-G(2 \xi))} k(\xi)(G(2 \xi)-G(\xi)) \frac{d \xi}{V(\xi)}$. On the other hand we obtain the same expression for $-\pi^{\prime}(\lambda)$ by differentiation of (4.4).

EXERCISE 4.7. Determine $\sigma(A)$ for $A$ from Example 2.7.

EXERCISE 4.8. Do the same for $A$ from Example 2.12 and for $B$ from Example 2.14.

\section{The characteristic equation}

Using the definition (1.5), (1.8), (3.4) and (4.4) we can rewrite $\pi(\lambda)$ as

$$
\pi(\lambda)=2 \int_{a}^{1} \frac{b(\xi)}{V(\xi)} \exp \left(-\int_{\xi / 2}^{\xi} \frac{\lambda+\mu(\eta)+b(\eta)}{V(\eta)} d \eta\right) d \xi .
$$

Since, $V, \mu$ and $b$ are nonnegative $\pi$ is strictly decreasing on the real axis. Clearly $\pi(-\infty)=+\infty$ and $\pi(+\infty)=0$. We conclude that there exists precisely one real root which we call $\lambda_{d}$ (d means dominant, a terminology explained in Remark 5.6 (i) below; note that in section I.4 we called the real root $k$; so $k=\lambda_{d}$ ). From the explicit expression for $\pi^{\prime}$ obtained by differentiation of $(5.1)$ it readily follows that $\pi^{\prime}\left(\lambda_{d}\right)<0$. We conclude from Theorem 4.6:

THEOREM 5.1. A has precisely one real eigenvalue $\lambda_{d}$ and this is a simple eigenvalue.

Since $V, \mu$ and $b$ are real valued $\overline{\pi(\lambda)}=\pi(\bar{\lambda})$, where the bar denotes complex conjugation. Hence non-real roots of $\pi(\lambda)=1$ occur in complex conjugate pairs. From the definition (5.1) it follows almost directly that $\pi$ is an analytic function. Hence the roots are isolated points, without any finite point of accumulation. In order to obtain further information about the position of the roots we try to write $\pi$ as a Laplace transform. Formula (4.4) suggests the transformation

$$
\tau=G(2 \xi)-G(\xi)
$$

Since

$$
\frac{d \tau}{d \xi}=\frac{2}{V(2 \xi)}-\frac{1}{V(\xi)}
$$

we need a condition on $V$ for (5.2) to define a one-to-one relationship. In the following we shall treat three cases:

DEFINITION 5.2 .

Case I : $V(2 x)<2 V(x), \frac{1}{2} a \leqslant x \leqslant \frac{1}{2}$,

Case II : $V(2 x)=2 V(x), \frac{1}{2} a \leqslant x \leqslant \frac{1}{2}$,

Case III : $\left\{\begin{array}{ll}V(2 x)=2 V(x), & \beta<x \leqslant \frac{1}{2}, \\ V(2 x)<2 V(x), & \frac{1}{2} a \leqslant x \leqslant \beta,\end{array}\right\}$ for some $\beta \in\left(\frac{1}{2} a, \frac{1}{2}\right)$. 
EXERCISE 5.3. Verify that the general solution of $V(2 x)=2 V(x), \frac{1}{2} a \leqslant x \leqslant \frac{1}{2}$, is given by $V(x)=x p(\ln x / \ln 2)$ with $p$ an arbitrary one-periodic function.

REMARKS 5.4. (i) Mathematically there is no difference between $V(2 x)<2 V(x)$ and $V(2 x)>2 V(x)$, for all $x$, but biologically the first seems reasonable and the second absurd.

(ii) The list of cases is far from exhaustive. Occasionaly we shall state remarks, exercises and results for still other cases. See Theorem 5.10 and the end of section 12, in particular Theorem 12.3.

THEOREM 5.5. In Case I there exists $\epsilon>0$ such that every root $\lambda \neq \lambda_{d}$ of the characteristic equation $\pi(\lambda)=1$ satisfies $\operatorname{Re} \lambda \leqslant \lambda_{d}-\epsilon$.

PROOF. Let $\xi(\tau)$ be the inverse function of $\tau(\xi)$ defined in (5.2). Then

$$
\pi(\lambda)=\int_{G(a)}^{G(1)-G\left(\frac{1}{2}\right)} e^{-\lambda \tau} \frac{k(\xi(\tau))}{V(\xi(\tau))} \frac{d \xi}{d \tau}(\tau) d \tau .
$$

Hence $\pi$ is of the form

$$
\pi(\lambda)=\int_{c_{1}}^{c_{2}} e^{-\lambda \tau} K(\tau) d \tau
$$

with $c_{2}>c_{1}>0$ and $K(\tau) \geqslant 0$ and not identically zero. Putting $\lambda=\mu+i \nu$ we obtain

$$
\pi(\lambda)=\int_{c_{1}}^{c_{2}} \cos \nu \tau e^{-\mu \tau} K(\tau) d \tau-i \int_{c_{1}}^{c_{2}} \sin \nu \tau e^{-\mu \tau} K(\tau) d \tau,
$$

from which we infer that for $\nu \neq 0$

$$
|\operatorname{Re} \pi(\lambda)|<\pi(\mu)=\pi(\operatorname{Re} \lambda) .
$$

Since $0<\pi(\mu) \leqslant 1$ for $\mu \geqslant \lambda_{d}$ necessarily $\operatorname{Re} \lambda<\lambda_{d}$ if $\lambda$ is a root. According to the Lemma of Riemann-Lebesgue (RUDIN, 1974, 5.14)

$$
\lim _{|\nu| \rightarrow \infty} \int_{c_{1}}^{c_{2}} \cos \nu \tau e^{-\mu \tau} K(\tau) d \tau=0
$$

uniformly for $\mu$ in compact sets. So in each vertical strip $\mu_{1} \leqslant \operatorname{Re} \lambda \leqslant \mu_{2}$ there can be at most finitely many roots. The conclusion of the theorem is now obvious.

REMARKS 5.6. (i) Since $\lambda_{d}$ is the eigenvalue with largest real part we call it the dominant eigenvalue.

(ii) One can use Hadamard's Factorization Theorem for entire functions of order one to show that there exist infinitely many roots. See chapter VIII of TITCHMARSH (1979).

THEOREM 5.7. In Case II the roots of $\pi(\lambda)=1$ are given explicitly by

$$
\lambda_{l}=\frac{1}{G(a)}\left\{\ln \int_{a / 2}^{1 / 2} \frac{k(\xi)}{V(\xi)} d \xi+2 l \pi i\right\}, l \in \mathbb{Z} .
$$

Proof. In this case $G(2 x)-G(x)=$ constant $=G(a)$ and consequently

$$
\pi(\lambda)=e^{-\lambda G(a)} \int_{a / 2}^{1 / 2} \frac{k(\xi)}{V(\xi)} d \xi
$$

Taking logarithms in the equation $\pi(\lambda)=1$ yields the result (recall that the "complex" logarithm is multi valued or see Exercise 7.5 below).

THEOREM 5.8. In Case III the conclusion of Theorem 5.5 holds.

Proof.

$$
\pi(\lambda)=e^{-\lambda G(a)} \int_{\frac{1}{2} a}^{\beta} \frac{k(\xi)}{V(\xi)} d \xi+\int_{G(2 \beta)-G(\beta)}^{G(1)-G\left(\frac{1}{2}\right)} e^{-\lambda \tau} \frac{k(\xi(\tau))}{V(\xi(\tau))} \frac{d \xi}{d \tau}(\tau) d \tau
$$


with $\xi(\tau)$ implicitly defined by (5.2). The proof of Theorem 5.5 carries over almost verbatim.

EXERCISE 5.9. Assume that $V(2 x)>2 V(x)$ for $\frac{1}{2} a \leqslant x<\beta$ and $V(2 x)<2 V(x)$ for $\beta<x \leqslant \frac{1}{2}$ for some $\beta \in\left(\frac{1}{2} a, \frac{1}{2}\right)$. Prove that the conclusion of Theorem 5.5 holds.

By now it should be clear that Case II is really exceptional and we summarize and extend our conclusions in:

THEOREM 5.10. If $V(2 x) \neq 2 V(x)$ for some $x \in\left[\frac{1}{2} a, \frac{1}{2}\right]$ the real eigenvalue $\lambda_{d}$ is strictly dominant. If $V(2 x)=2 V(x)$ for all $x \in\left[\frac{1}{2} a, \frac{1}{2}\right]$ then, on the contrary, there exist countably many eigenvalues on the line $\operatorname{Re} \lambda=\lambda_{d}$, which form an additive subgroup of this line. In all cases the eigenvector corresponding to $\lambda_{d}$ is positive.

In section 13 we shall put these findings in the right perspective and there we also present several important references.

\section{Decomposition of the population state space $X$}

Let $\psi_{d}$ denote the eigenvector corresponding to the real eigenvalue $\lambda_{d}$. Then

$$
T(t) \psi_{d}=e^{\lambda_{d} t} \psi_{d}
$$

EXERCISE 6.1. Prove this identity. Hint: compute $\frac{d}{d t} T(t) \psi_{d}$ using Theorem 2.5.

An obvious conjecture is now that for arbitrary $\phi \in X$ the $X$-valued function $T(t) \phi$ will have for $t \rightarrow \infty$ its fastest growth "in the direction" of $\psi_{d}$ when $\lambda_{d}$ strictly leads the field of real parts of eigenvalues of $A$. To begin with we have to give a precise meaning to "in the direction".

The eigenvector $\psi_{d}$ spans the linear subspace $\Re\left(\lambda_{d} I-A\right)$ which clearly is invariant under $\{T(t)\}$. Our plan is to decompose $X$ into $\vartheta\left(\lambda_{d} I-A\right)$ and another invariant subspace and to prove subsequently that the restriction of $\{T(t)\}$ to that second subspace obeys an exponential estimate with exponent $\lambda_{d}-\epsilon$ when $\operatorname{Re} \lambda<\lambda_{d}-\epsilon$ for all eigenvalues $\lambda$ other than $\lambda_{d}$. In this section we carry out the first and easiest half of the plan only, postponing the second half to sections 8 and 9 .

Defrnirion 6.2. Let $X$ be a Banach space. A bounded linear operator $P: X \rightarrow X$ is called a projection if and only if $P^{2}=P$.

Definimion 6.3. $X$ is the direct sum of two linear subspaces $Y$ and $Z$ if and only if for each $x \in X$ there exist a unique $y \in Y$ and $z \in Z$ such that $x=y+z$. NOTATXON: $X=Y \oplus Z$.

EXERCISE 6.4. Let $P: X \rightarrow X$ be a projection. Show that $X=\Re(P) \oplus \Re(P)$ and that $\Re(P)=\Re(I-P)$. Show that $\Re(P)$ and $\Re(P)$ are closed.

EXERCISE 6.5. Let $X=Y \oplus Z$ with $Y$ and $Z$ closed linear subspaces. Define linear operators $P, Q: X \rightarrow X$ by $P x=y$ and $Q x=z$ where $y \in Y$ and $z \in Z$ are defined by $x=y+z$. Show that $P$ and $Q$ are projections and that $Q=I-P$. (Hint: In order to show that $P$ and $Q$ are bounded one can use the closed graph theorem: If the linear operator $L: X_{1} \rightarrow X_{2}$ is closed then $L$ is continuous; see Definition 2.3 for the notion of a closed operator).

The next theorem is one of the key-stones of our approach. It shows that one can associate with spectral values which are poles of the resolvent a natural direct sum decomposition of the underlying space. For the proof we refer to YosIDA, 1980, VIII.8.

THEOREM 6.6. Let $L$ be a closed linear operator on the complex Banach space $X$ and let $\lambda_{0}$ be an isolated point of $\sigma(L)$. Then $\lambda_{\leftrightarrow}(\lambda I-L)^{-1}$ is a holomorphic mapping (in a punctured neighbourhood $\Omega \backslash\left\{\lambda_{0}\right\}$ of $\lambda_{0}$ ) admitting the Laurent expansion

$$
(\lambda I-L)^{-1}=\sum_{-\infty}^{+\infty}\left(\lambda-\lambda_{0}\right)^{k} A_{k}
$$


where for each $k \in \mathbb{Z}$

$$
A_{k}=\frac{1}{2 \pi i} \int_{\Gamma}\left(\lambda-\lambda_{0}\right)^{-k-1}(\lambda I-L)^{-1} d \lambda
$$

with $\Gamma$ a (counter-clockwise oriented) circumference $\left|\lambda-\lambda_{0}\right|=\eta$, where $\eta$ is so small that the circle $\left|\lambda-\lambda_{0}\right| \leqslant \eta$ does not contain singularities of $(\lambda I-L)^{-1}$ other than $\lambda_{0}$ itself. The operator $A_{-1}$ is a projection on $X$. $k \geqslant m$

If $\lambda_{0}$ is a pole of $(\lambda I-L)^{-1}$ of order $m$ (i.e., $A_{-m} \neq 0$ and $A_{k}=0$ for $k<-m$ ) then $\lambda_{0}$ is an eigenvalue of $L$ and for

$$
\begin{aligned}
& \Re(A-1)=\Re\left(\left(\lambda_{o} I-L\right)^{k}\right) \\
& \Re(I-A-1)=\Re\left(\left(\lambda_{0} I-L\right)^{k}\right)
\end{aligned}
$$

so that, in particular,

$$
X=\Im\left(\left(\lambda_{0} I-L\right)^{m}\right) \oplus \Re\left(\left(\lambda_{0} I-L\right)^{m}\right)
$$

REMARK 6.7. Calculations involving integrals of complex variable operator-valued functions can be performed in precisely the same way as calculations involving ordinary complex functions. Thus the expression (6.3) for $A_{k}$ is obtained from the Cauchy formula. To determine $A_{-1}$ in practice one simply calculates the "coefficient" of $\left(\lambda-\lambda_{0}\right)^{-1}$ in the expansion of $(\lambda I-L)^{-1}$ in powers of $\lambda-\lambda_{0}$ (see below for a concrete example).

We are now going to apply Theorem 6.6 to the operator $A$. The calculations in section 4, notably formulas $(4.2)$ (4.7) imply that

$$
\left((\lambda I-A)^{-1} f\right)(x)=(1-\pi(\lambda))^{-1} \zeta(\lambda, f) \Psi(\lambda, x)+R(\lambda, f, x)
$$

where

$$
\Psi(\lambda, x)= \begin{cases}e^{\lambda\left(G\left(\frac{1}{2}\right)-G(x)\right)} & , \frac{1}{2} \leqslant x \leqslant 1, \\ \int_{a / 2}^{x} e^{\lambda\left(G(\xi)-G(2 \xi)-G(x)+G\left(\frac{1}{2}\right)\right\}} \frac{k(\xi)}{V(\xi)} d \xi & , \frac{1}{2} a \leqslant x \leqslant \frac{1}{2},\end{cases}
$$

and where $\lambda \mapsto R(\lambda, f, x)$ is analytic. Hence the singularities of $\lambda_{\mapsto}(\lambda I-A)^{-1}$ are precisely the zeros of $1-\pi(\lambda)$ and these are poles. The order of the pole equals the order of the zero. In the case of $\lambda_{d}$ we have $\pi^{\prime}\left(\lambda_{d}\right) \neq 0$ and the order is one (cf. Theorem 5.1). The residue of $\lambda_{r} \rightarrow\left((\lambda I-A)^{-1} f\right)(x)$ in $\lambda=\lambda_{d}$ is given by

$$
\frac{\zeta\left(\lambda_{d}, f\right)}{-\pi^{\prime}\left(\lambda_{d}\right)} \Psi\left(\lambda_{d}, x\right)=\frac{\zeta\left(\lambda_{d}, f\right)}{-\pi^{\prime}\left(\lambda_{d}\right)} \psi_{d}(x)
$$

so the projection $A_{-1}$, here denoted by $P$, is given by

$$
P f=\frac{\zeta\left(\lambda_{d}, f\right)}{-\pi^{\prime}\left(\lambda_{d}\right)} \psi_{d}
$$

EXERCISE 6.8. Verify that $P$ is a projection using the calculations in the proof of Theorem 4.6.

COROLlaRy 6.9. $X=\Re\left(\lambda_{d} I-A\right) \oplus \Re\left(\lambda_{d} I-A\right)$ and the corresponding projection onto $\Re\left(\lambda_{d} I-A\right)$ is $P$ defined in $(6.7)$.

EXERCISE 6.10. Verify that $\mathfrak{R}\left(\lambda_{d} I-A\right)$ is invariant under $\{T(t)\}$ and show that $P$ commutes with $T(t)$.

\section{Relations between the spectra of $A$ and of $T(t)$}

In this and the following section $X$ denotes a Banach space, $\{T(t)\}$ a strongly continuous semigroup of bounded linear operators on $X$ and $A$ the infinitesimal generator of $\{T(t)\}$. We intend to explore the conclusions about the behaviour of $\{T(t)\}$ (especially for large $t$ ), that can be drawn from our knowledge of the spectrum of $A$. In this section we concentrate on the spectrum of $T(t)$ and in the next one we shall consider exponential estimates.

Notation: If $W$ is a subset of $\mathbb{C}$ then $e^{t W}=\left\{e^{t w} \mid w \in W\right\}$. 
TheOREM 7.1. $e^{t \sigma(A)} \subset \sigma(T(t)), t \geqslant 0$.

The proof will be delegated to the next two exercises.

EXERCISE 7.2. Define $Z(t) \phi=\int_{0}^{t} e^{\lambda(t-\tau)} T(\tau) \phi d \tau$. Show that

(i) $\Re(Z(t)) \subset \mathscr{D}(A)$;

(ii) $A Z(t) \phi=Z(t) A \phi, \forall \phi \in \mathscr{D}(A)$;

(iii) $(\lambda I-A) Z(t)=e^{\lambda t} I-T(t)$.

EXERCISE 7.3. Prove Theorem 7.1 by showing that for $e^{\lambda t} \in \rho(T(t))$ the operator $Z(t)\left(e^{\lambda t} I-T(t)\right)^{-1}$ is the inverse of $\lambda I-A$.

EXERCISE 7.4. Assume that $e^{-\lambda t}\|T(t)\| \rightarrow 0$ for $t \rightarrow \infty$ and $\operatorname{Re} \lambda>\omega_{0}$ (in the next section we prove that one can always find $\omega_{0}$ such that this holds). Define $R(\lambda)=\int_{0}^{\infty} e^{-\lambda \tau} T(\tau) d \tau$ for $\operatorname{Re} \lambda>\omega_{0}$. Show that $\Re(R(\lambda)) \subset \mathcal{D}(A)$ and that $(\lambda I-A) R(\lambda)=I$. The observation that $A$ and $R(\lambda)$ commute on $\mathscr{D}(A)$ implies that $R(\lambda)$ is the resolvent of $A$ for $\operatorname{Re} \lambda>\omega_{0}$. Note the analogy with the identity

$$
\int_{0}^{\infty} e^{-\lambda \tau} e^{\alpha \tau} d \tau=(\lambda-\alpha)^{-1} .
$$

EXERCISE 7.5. (For those who have little experience with complex exponentials and logarithms). For fixed $t>0$, analyse the mapping $\lambda_{\mapsto e^{\lambda t}}$ (from $\mathbb{C}$ into $\mathbb{C}$ ) and its multivalued inverse. Concentrate in particular on vertical and horizontal lines and their images. (Draw each line in some colour and draw its image in the same colour .) Use the periodicity with respect to the imaginary part of $\lambda$ as a motivation to divide the plane in horizontal strips

$$
\left\{\lambda \mid(2 l-1) \frac{\pi}{t}<\operatorname{Im} \lambda \leqslant(2 l+1) \frac{\pi}{t}\right\}, l \in \mathbb{Z} .
$$

One can show by means of examples (see, for instance, Example 8.6) that the converse of Theorem 7.1 does not hold. The situation is more surveyable if we restrict our attention to the point spectrum.

Theorem 7.6. $e^{t P \sigma(A)} \subset P \sigma(T(t)) \subset\left[e^{t P \sigma(A)} \cup\{0\}\right]$; more precisely we have that $e^{\lambda t} \in P \sigma(T(t))$ if $\lambda \in P \sigma(A)$ and that for at least one $l \in \mathbb{Z}, \lambda+\frac{2 \pi i l}{T} \in P \sigma(A)$ if $e^{\lambda t} \in P \sigma(T(t))$.

EXERCISE 7.7. Prove the first inclusion by means of the identity (on $\mathscr{D}(A)) e^{\lambda t} I-T(t)=(\lambda I-A) Z(t)=Z(t)(\lambda I-A)$.

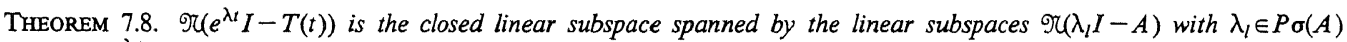
such that $e^{\lambda_{t} t}=e^{\lambda t}$.

EXERCISE 7.9. Let $\phi \in \mathscr{T}\left((\lambda I-A)^{2}\right)$. Show that

$$
T(t) \phi=e^{\lambda t} \phi+t e^{\lambda t}(A-\lambda I) \phi
$$

and deduce from this identity that $\phi \in \mathscr{T}\left(\left(e^{\lambda t} I-S(t)\right)^{2}\right)$ while

$\phi \in \mathscr{T}\left(e^{\lambda t} I-T(t)\right)$ iff $\phi \in \mathscr{T}(\lambda I-A)$.

EXERCISE 7.10. Prove by induction that $\vartheta\left((\lambda I-A)^{k}\right) \subset \vartheta\left(\left(e^{\lambda t} I-S(t)\right)^{k}\right)$.

REMARK 7.11. NUSSBAUM (1984) proves the analogue of Theorem 7.8 for the $k$-times iterated operators. A spectral mapping theorem similar to Theorem 7.6 holds for the residual spectrum, but the continuous spectrum of the semigroup need not be faithful to that of the generator.

\section{Exponential estimates}

Aiming at exponential estimates we shall study the large time behaviour of $\frac{1}{t} \log \|T(t)\|$. It will appear that this 
function has a limit for $t \rightarrow \infty$. The next and far more difficult problem is to characterize the limit in terms of (known) properties of the generator $A$ and we will find that certain technical (so-called compactness) conditions are very helpful if not essential. To begin with we introduce an important concept.

Let $L$ be a bounded linear operator on $X$. The spectral radius $r_{\sigma}(L)$ can be defined by

$$
r_{\mathrm{o}}(L)=\inf _{k \geqslant 1}\left\|L^{k}\right\|^{\frac{1}{k}}
$$

although it clearly deserves its name because an alternative definition is provided by point (ii) of:

\section{LEMMA 8.1.}

(i) $\lim _{k \rightarrow \infty}\left\|L^{k}\right\|^{\frac{1}{k}}$ exists and equals $r_{\sigma}(L)$

(ii) $\sup _{\lambda \in \sigma(L)}|\lambda|=r_{\sigma}(L)$.

The proof of (i) is based on a discrete version of the following auxiliary result which we need below.

LEMMA 8.2. Let $p:[0, \infty) \rightarrow \mathbb{R}$ be bounded on each finite subinterval and subadditive (i.e. $\left.p\left(t_{1}+t_{2}\right) \leqslant p\left(t_{1}\right)+p\left(t_{2}\right), \forall t_{1, t_{2}} \geqslant 0\right)$. Define $\nu=\inf _{t>0} \frac{p(t)}{t}$. Then $\lim _{t \rightarrow \infty} \frac{p(t)}{t}$ exists and equals $\nu$.

We want to apply this lemma with $p(t)=\log \|T(t)\|$ and therefore we need the following:

LEMMA 8.3. $\|T(t)\|$ is bounded on bounded intervals.

This lemma is a straightforward consequence of the uniform boundedness principle (the Banach-Steinhaus theorem, see RUDIN, 1974, 5.8).

We now define

$$
\omega_{0}=\omega_{0}(T(t))=\inf _{t>0} \frac{1}{t} \log \|T(t)\|
$$

and find

\section{THEOREM 8.4 .}

i) $\lim _{t \rightarrow \infty} \frac{1}{t} \log \|T(t)\|$ exists and equals $\omega_{0}$.

ii) $\forall \omega>\omega_{0} \exists M(\omega)$ such that $\|T(t)\| \leqslant M(\omega) e^{\omega t}, t \geqslant 0$.

iii) $r_{o}(T(t))=e^{\omega_{0} t}$ for $t \geqslant 0$.

EXERCISE 8.5. Prove points (ii) and (iii).

It remains to characterize the so-called growth bound $\omega_{0}$. The following example (due to GREINER, VorgT \& WOLFF, 1981) shows that the obvious conjecture $\omega_{0}=s(A)$, where by definition

$$
s(A)=\sup \{\operatorname{Re} \lambda \mid \lambda \in \sigma(A)\},
$$

is false.

EXAMPLE 8.6. Define $\|f\|_{1}=\int_{0}^{\infty} e^{\tau}|f(\tau)| d \tau$ and let for some $p \in(1, \infty)$

$$
X=\left\{f \in L_{p}(0, \infty) \mid\|f\|_{1}<\infty\right\}
$$

provided with the norm $\|f\|=\|f\|_{L}+\|f\|_{1}$. Then $X$ is a Banach space and the translation semigroup $(T(t) f)(x)=f(x+t), t \geqslant 0$, is strongly continous. Clearly $\|T(t)\| \leqslant 1$. In order to show that $\|T(t)\|=1$ we introduce

$$
f_{i}(\tau)= \begin{cases}1 & \text { for } t \leqslant \tau \leqslant t+e^{p}, \\ 0 & \text { elsewhere }\end{cases}
$$


A simple calculation shows that

$$
\left\|f_{\epsilon}\right\|=\epsilon+\epsilon^{t}\left(e^{\ell}-1\right) \text { and }\left\|T(t) f_{\epsilon}\right\|=\epsilon+e^{\ell}-1
$$

so that

$$
\left\|T(t) f_{\epsilon}\right\|=\left\|f_{\mathrm{c}}\right\|(1-o(1)) \text { for } € \downarrow 0 .
$$

We conclude that $\|T(t)\|=1$ and $\omega_{0}=0$.

The infinitesimal generator is $A u=u^{\prime}$ with $\mathscr{D}(A)=\left\{u \mid u\right.$ is absolutely continuous and $\left.u^{\prime} \in X\right\}$. We shall demonstrate that $\{\lambda \mid \operatorname{Re} \lambda>-1\} \subset \rho(A)$. The abstract equation $(\lambda I-A) u=f$ leads to $\lambda u-u^{\prime}=f$ and hence to

$$
u(t)=\int_{0}^{\infty} e^{-\lambda \tau} f(t+\tau) d \tau=e^{\lambda t} \int_{t}^{\infty} e^{-\lambda \tau} f(\tau) d \tau
$$

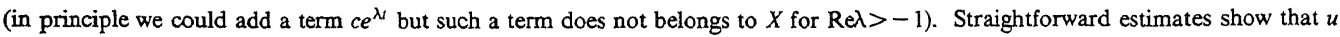
thus defined belongs to $X$ for $\operatorname{Re} \lambda>-1$ and consequently $u^{\prime}=\lambda u-f \in X$. We conclude that $u=(\lambda I-A)^{-1} f$ and that $\lambda \in \rho(A)$. Finally, $t_{\mapsto \rightarrow e^{\lambda t}}$ is an eigenvector corresponding to the eigenvalue $\lambda$ if $\operatorname{Re} \lambda<-1$. Hence $\sup _{\lambda \in \sigma(A)} \operatorname{Re} \lambda=-1 \neq 0=\omega_{0}$.

In view of Theorem 7.6 we know that $\omega_{0}=\sup \{\operatorname{Re} \lambda \mid \lambda \in \sigma(A)\}$ whenever $T(t)$ has, for some $t>0$, an eigenvalue on the circumference $|z|=r_{\sigma}(T(t))=e^{\omega_{0} t}$. Such is certainly the case if all spectral values, except possibly $z=0$, are eigenvalues. An important class of operators, viz. compact operators, has this property. We need some terminology.

A subset $W$ of $X$ is called compact if every cover of $X$ by open sets contains a finite subcover. An equivalent but more imaginitive condition for compactness is that every sequence in $W$ has a subsequence that converges to an element of $W$ (N.B. The equivalence holds for metric spaces and therefore certainly for Banach spaces; see HuTson \& PYM, 1980). $W$ is called precompact (or, relatively compact) if the closure $\bar{W}$ of $W$ is compact.

The compact subsets of $\mathbb{R}^{N}$ are precisely the bounded, closed sets. If $X$ is some space of functions defined on a domain $\Omega \subset \mathbb{R}^{N}$ one can sometimes find a reasonable simple criterion for the (pre)compactness of subsets of $X$ (see KUFNER, JOHN \& FUCIK, 1977). We present one well-known example which we need later on.

Let $\Omega$ be a compact subset of $\mathbb{R}^{N}$. A set $W$ of continuous functions on $\Omega$ is called uniformly bounded if it is a bounded subset of $C(\Omega)$ (i.e., there exists a constant $K$ such that $\|f\|=\sup _{x \in \Omega}|f(x)| \leqslant K$ for all $f \in W$ ). The set $W$ is called equicontinuous if $\forall \epsilon>0 \exists \delta=\delta(\epsilon)>0$ such that $\forall f \in W$ and $\forall x, y \in \Omega$ with $|x-y|<\delta$ the estimate $|f(x)-f(y)|<\epsilon$ holds.

THEOREM 8.7. (Arzela-Ascoli) A subset $W$ of $C(\Omega)$ is precompact if and only if $W$ is uniformly bounded and equicontinuous.

A linear operator is called compact (or completely continuous) if bounded sets are mapped onto precompact sets. Again there is an equivalent condition: the image of any bounded sequence should contain a convergent subsequence. It is not difficult to prove that compact linear operators are necessarily bounded and that the compact linear operators form a closed linear subspace of the space of bounded linear operators (provided with the operator norm). Moreover, the product of a compact and a bounded operator is compact.

THEOREM 8.8. Suppose $\operatorname{dim} X=\infty$ and let $L: X \rightarrow X$ be a compact linear operator. Then $0 \in \sigma(L)$. ( 0 can belong to $P \sigma(L), R \sigma(L)$ or $C \sigma(L))$ and $\sigma(L) \backslash\{0\}$ consists of either a finite number of eigenvalues or an infinite sequence of eigenvalues that converges to zero.

COROLLARY 8.9. Suppose that $T\left(t_{0}\right)$ is compact for some $t_{0}>0$. Then $\omega_{0}=s(A)=\sup \{\operatorname{Re} \lambda \mid \lambda \in P \sigma(A)\}$.

REMARK. The semigroup property implies that $T(t)$ is compact for $t \geqslant t_{0}$ whenever $T\left(t_{0}\right)$ is compact. Indeed, $T(t)=T\left(t_{0}\right) T\left(t-t_{0}\right)$ and the product of a compact and a bounded operator is compact.

Although one can use Corollary 8.9 in many applications it is not strong enough to cover many others. We need some refinements.

The (Kuratowski) measure of noncompactness $\alpha(W)$ of a bounded set $W \subset X$ is the infimum of the positive numbers $d$ for which $W$ can be covered by finitely many sets of diameter less than or equal to $d$ (recall that the diameter of a set $U$ is the supremum of $\{\|x-y\| \mid x, y \in U\}$; note that $W$ is compact iff $\alpha(W)=0)$. The measure of noncompactness $|L|_{\alpha}$ of a bounded linear operator $L$ is the infimum of the positive numbers $\theta$ for which $\alpha(L(W)) \leqslant \theta \alpha(W)$ for all bounded sets $W \subset X$ (hence $L$ is compact iff $|L|_{\alpha}=0 ;||_{\alpha}$ defines a seminorm on $X$, cf. NussBaUM, (1970).

The (Browder) essential spectrum $\sigma_{e}(L)$ of a closed operator $L$ is defined as the set of those $\lambda \in \sigma(L)$ for which at 
least one of the following conditions is satisfied

(i) $\Re(\lambda I-L)$ is not closed;

(ii) $\lambda$ is an accumulation point of $\sigma(L)$;

(iii) the generalized eigenspace corresponding to $\lambda$ is infinite-dimensional.

It is known that the complement $\sigma(L) \backslash \sigma_{e}(L)$ consists of isolated poles of finite order of the resolvent. The elements of $\sigma(L) \backslash \sigma_{e}(L)$ are called normal eigenvalues. Finally, we define the essential spectral radius

$$
r_{e}(L)=\sup \left\{|\lambda| \mid \lambda \in \sigma_{e}(L)\right\}
$$

and quote the following result of NUSSBAUM (1970):

LEMMA 8.10. $r_{e}(L)=\lim _{k \rightarrow \infty}\left|L^{k}\right|_{\alpha}^{\frac{1}{k}}=\inf _{k \geq 1}\left|L^{k}\right|_{\alpha}^{\frac{1}{k}}$

The idea to use these concepts and results in the context of linear semigroups seems to be due to PRUss (1981). Detailed proofs of the following theorems can be found in WEBB (1985a). In analogy with the definition of $\omega_{0}$ in (8.2) we introduce

$$
\omega_{e}=\omega_{e}(T(t))=\inf _{t>0} \frac{1}{t} \log |T(t)|_{\alpha}
$$

(with the convention that $\log 0=-\infty$ ).

THEOREM 8.11 .

i) $\lim _{t \rightarrow \infty} \frac{1}{t} \log |T(t)|_{\alpha}$ exists and equals $\omega_{e}$

ii) $r_{e}(T(t))=e^{\omega_{e} t}, t>0$ (with the convention $e^{-\infty}=0$ )

\section{THEOREM 8.12 .}

$$
\omega_{0}=\max \left\{\omega_{e}, \omega_{n}\right\} \text { where } \omega_{n}=\sup \{\operatorname{Re} \lambda \mid \lambda \text { is a normal eigenvalue of } A\}
$$

So, provided we can show (using Theorem 8.11) that $\omega_{e}<\omega_{n}$, we have obtained a characterization of $\omega_{0}$ in terms of the spectrum of $A$.

REMARK 8.13. Using Theorem 8.12 one can show that $\omega_{0}=\max \left\{\omega_{e}, s(A)\right\}$

\section{The stable size distribution}

Let us return to our concrete example, the semigroup $\{T(t)\}$ and the generator $A$ that go with the model for cell proliferation. The first thing we try is to prove that the semigroup is compact after finite time. Since the semigroup is constructively defined by the generation expansion we shall scrutinize the terms in this expansion.

The zero'th generation

$$
\left(U_{0}(t) \phi\right)(x)=\phi\left(G^{-1}(G(x)-t)\right)
$$

transforms and translates the initial function $\phi$ without changing the smoothness, and a glance at the Arzela-Ascoli Theorem 8.7 should suffice to conclude that $U_{0}(t)$ is not compact for $t<G(1)$. For $t \geqslant G(1)$, however, $U_{0}(t)$ is the zero-operator and thus certainly compact.

The first generation corresponds to the operator

$$
U_{1}(t) \phi=\int_{0}^{t} U_{0}(t-\tau) C U_{0}(\tau) \phi d \tau .
$$

EXERCISE 9.1. Show that explicitly

$$
\left(U_{1}(t) \phi\right)(x)=\int_{0}^{t} k(X(-t+\tau, x)) \phi(X(-\tau, 2 X(-t+\tau, x))) d \tau
$$

where (cf. Exercise 3.3) 


$$
X(-t, x)=G^{-1}(G(x)-t)
$$

and where, for convenience, $G(x)=G(1)$ for $x \geqslant 1$ and $k(x)=0$ for $x>\frac{1}{2}$.

EXERCISE 9.2. Let $\xi(\tau, t, x)=G(X(-\tau, 2 X(-t+\tau, x)))=G(2 X(-t+\tau, x))-\tau=G\left(2 G^{-1}(G(x)-t+\tau)\right)$. Show that

$$
\frac{\partial \xi}{\partial \tau}=\frac{2 V(X(-t+\tau, x))}{V(2 X(-t+\tau, x))}-1
$$

In order to prove compactness of $U_{1}(t)$ we try to rewrite (9.3) such that the argument of $\phi$ does not contain the variable $x$ anymore (indeed, we need equicontinuity without knowing more about $\phi$ than some sup-norm bound). Formula (9.5) implies that we can do so provided we restrict $V$ to Case I of Definition 5.2:

Assumption 9.3: For the rest of this section we assume that $V(2 x)<2 V(x)$, for $\frac{1}{2} a \leqslant x \leqslant \frac{1}{2}$.

Combining the results of Exercises 9.1 and 9.2 we have

$$
\left(U_{1}(t) \phi\right)(x)=\int_{G(2 X(-t, x))}^{G(2 x)-t} k(X(-t+\tau(\xi, t, x))) \phi\left(G^{-1}(\xi)\right) \frac{\partial \tau}{\partial \xi}(\xi, t, x) d \xi
$$

where $\tau(\xi, t, x)$ is the inverse function of $\xi(\tau, t, x)$ defined in Exercise 9.2. From this representation one can prove that $U_{1}(t)$ is compact, but the proof is rather technical. The essential ideas can be conveniently demonstrated in the simple special case $V(x) \equiv 1$.

EXERCISE 9.4. Show that (9.6) reduces to

$$
\left(U_{1}(t) \phi\right)(x)=\int_{2 x-2 t}^{2 x-t} k(t-x+\xi) \phi(\xi) d \xi
$$

with the convention $k(x)=0$ for $x \leqslant \frac{1}{2} a$ and $x>\frac{1}{2}$, when $V(x) \equiv 1$.

THEOREM 9.5. Under Assumption 9.3 the first generation operator $U_{1}(t)$ is compact for all $t \geqslant 0$.

Proof (for $V(x) \equiv 1$ ). For any $y>x$ we obtain from (9.7) that

$$
\left|\left(U_{1}(t) \phi\right)(x)-\left(U_{1}(t) \phi\right)(y)\right| \leqslant((1)+(2)+(3))\|\phi\|
$$

where

$$
\begin{aligned}
& \text { (1) }=\int_{2 x-2 t}^{2 x-t}|k(t-x+\xi)-k(t-y+\xi)| d \xi \leqslant \int_{-\infty}^{\infty}|k(\tau)-k(\tau+x-y)| d \tau \\
& \text { (2) }=\int_{2 x-2 t}^{2 y-2 t} k(t-y+\xi) d \xi=\int_{2 x-y-t}^{y-t} k(\tau) d \tau \\
& \text { (3) }=\int_{2 x-t}^{2 y-t} k(t-y+\xi) d \xi=\int_{2 x-y}^{y} k(\tau) d \tau
\end{aligned}
$$

As $y-x \downarrow 0$, (1) goes to zero since translation is continuous in $L_{1}(\mathbb{R})$ (which is easy to prove using the fact that $C^{\infty}$. functions with compact support are dense in $L_{1}(\mathbb{R})$ ) and (2) and (3) go to zero since the indefinite integral of an $L_{1^{-}}$function is continuous (even absolutely continuous, see Definition 2.8 and Theorem 2.9). We conclude that $\left\{U_{1}(t) \phi \mid\|\phi\| \leqslant K\right\}$ is equicontinuous. Since $\left|\left(U_{1}(t) \phi\right)(x)\right| \leqslant \int_{\frac{1}{2} a}^{\frac{1}{2}} k(\tau) d \tau\|\phi\|$ this set is also uniformly bounded.

We next define inductively the $l^{\text {th }}$ generation operator

$$
U_{l}(t)=\int_{0}^{t} U_{0}(t-\tau) C U_{l-1}(\tau) d \tau
$$

Exactly the same type of arguments (note that in the proof of Theorem 9.5 the equicontinuity is uniform for $t \in[0, s]$ for any $s<\infty$ ) yield 
THEOREM 9.6. Under Assumption 9.3 the $l^{\text {th }}$ generation operator $U_{l}(t)$ is compact for all $t \geqslant 0$ and all $l \in \mathbb{N}$.

As an aside we remark that a short proof applies when $C$ is bounded (i.e., $k\left(\frac{1}{2}\right)=0$ ): the (Riemann) integral is a limit of finite sums and the set of compact linear operators is closed.

COROLlaRY 9.7. Under Assumption 9.3 the semigroup

$$
T(t)=\sum_{l=0}^{\infty} U_{l}(t)
$$

is compact for $t \geqslant G(1)$.

Proof. Only finitely many terms of the series are non-zero (see Lemma 3.8) so we don't have to worry about the sense of convergence.

EXERCISE 9.8. If $V(2 x)=2 V(x)$ for $\frac{1}{2} a \leqslant x \leqslant \frac{1}{2}$ then $\frac{\partial \xi}{\partial \tau}=0$ and consequently $\xi(\tau, t, x)=\xi(t, t, x)=$ $G(X(-t, 2 x))=G(2 x)-t$. Show that now

$$
\left(U_{1}(t) \phi\right)(x)=\phi\left(G^{-1}(G(2 x)-t)\right) \int_{0}^{t} k\left(G^{-1}(G(x)-t+\tau)\right) d \tau
$$

and convince yourself that $U_{\mathrm{l}}(t)$ will be compact only when the first generation has become extinct, i.e. for $t \geqslant G(2)$. Inductively one shows that $U_{l}(t)$ is not compact before $t=G(l+1)$ and therefore $T(t)$ will never be compact.

EXERCISE 9.9. Compute $G^{-1}(G(2 x)-t)$ for the special case $V(x)=x$.

EXERCISE 9.10. Compute $U_{2}(t)$ for the special case $V(x)=x$.

EXERCISE 9.11. Show that $G(2 x)-G(x)=$ constant $=G(a)$ when $V(2 x)=2 V(x)$ and give a biological interpretation of this identity.

We are now ready to reap the fruits of our efforts in sections $5-8$. In particular we are going to apply Corollary 8.9 to the restriction of $T(t)$ to the invariant subspace $\Re\left(\lambda_{d} I-A\right)$ (see Corollary 6.9 and Exercise 6.10). Denoting this restriction by $T_{R}(t)$ we observe that its generator is $A_{R}$, the restriction of $A$ to $R\left(\lambda_{d} I-A\right)$. Since $\sigma\left(A_{R}\right)=\sigma(A) \backslash\left\{\lambda_{d}\right\}$ we conclude from Theorem 5.5 that $\sup \left\{\operatorname{Re} \lambda \mid \lambda \in \sigma\left(A_{R}\right)\right\}<\lambda_{d}-\epsilon$ for some $\epsilon>0$ and subsequently from Corollary 8.9 that $\omega_{0}\left(T_{R}(t)\right)<\lambda_{d}-\epsilon$ where $\omega_{0}\left(T_{R}(t)\right)$ denotes the growth bound of $T_{R}(t)$, and, finally, from Theorem 8.4 (ii) that

$$
\left\|T_{R}(t)\right\| \leqslant M e^{\left(\lambda_{d}-c\right) t}, t \geqslant 0 .
$$

Recalling that $P$, defined by (6.7), denotes the projection onto $\mathscr{R}\left(\lambda_{d} I-A\right)$ along $\Re\left(\lambda_{d} I-A\right)$, we write

$$
T(t) \phi=T(t)(P \phi+(I-P) \phi)=e^{\lambda_{d} t} P \phi+T_{R}(t)(I-P) \phi=e^{\lambda_{d} t}\left(P \phi+O\left(e^{-\epsilon t}\right)\right), t \rightarrow \infty,
$$

and summarize our conclusions as one of the main results of this chapter.

THEOREM 9.12. (The stable size distribution). Under Assumption 9.3

$$
T(t) \phi=e^{\lambda_{d} t}\left(-\frac{\zeta\left(\lambda_{d}, \phi\right)}{\pi^{\prime}\left(\lambda_{d}\right)} \psi_{d}+O\left(e^{-\epsilon t}\right)\right), t \rightarrow \infty,
$$

or, in words: the dominant term in the asymptotic expansion of $T(t) \phi$ for $t \rightarrow \infty$ is the product of three factors

i) $e^{\lambda_{d} t}$ : an exponential function of time with exponent $\lambda_{d}$, the dominant eigenvalue

ii) $\psi_{d}$ : a fixed element of $X$, the eigenvector of $A$ corresponding to $\lambda_{d}$

iii) $-\frac{\zeta\left(\lambda_{d}, \phi\right)}{\pi^{\prime}\left(\lambda_{d}\right)}$ : a time-independent scalar which is the only factor that depends on the specific initial condition $\phi$

and the remainder terms are relatively exponentially smaller with an exponent that is determined by the distance along the real axis of $\lambda_{d}$ and the other eigenvalues of $A$.

EXERCISE 9.13. The eigenfunction $\psi_{d}$ corresponding to $\lambda_{d}$ is defined by (4.2) $-(4.3)$ with $\lambda=\lambda_{d}, f(x) \equiv 0$ and, say, $C=1$ as a normalization. Thus $\psi_{d}$ is a positive function. Show that $\zeta\left(\lambda_{d}, \phi\right)>0$ whenever $\phi \geqslant 0$ and $\phi \equiv 0$. 
We conclude that the cell population will grow exponentially when $\lambda_{d}>0$ (respectively, die out exponentially when $\lambda_{d}<0$ or approach a constant level when $\lambda_{d}=0$ ) while at the same time the (normalized, transformed) size distribution converges to a fixed distribution $\psi_{d}$ that does not depend on the initial condition. For that reason one calls $\psi_{d}$ the (transformed) stable size distribution. We refer back to section 1.4 for a discussion of the biological information that is contained in this result.

The techniques of this section required that we restricted our attention to the (biologically most important) Case I of Definition 5.5 (see Assumption 9.3). Our next objective will be to analyse the asymptotic behaviour of $T(t)$ in Case II: $V(2 x)=2 V(x), \frac{1}{2} a \leqslant x \leqslant 1$. In order to understand what happens in that case we need an auxiliary result (the difference equation (10.18) below) which can be obtained by a very important and useful technique called integration along characteristics.

\section{Interlude: integration along characteristics}

In this section we shall introduce and illustrate the technique of integration along characteristics by analysing in detail its application to the cell proliferation model. In Chapter III, section 4 we return to it in a more general context and there we explain some of its features in a more geometric language. Here we concentrate on those aspects that involve straightforward systematic calculations, repeating to some extent our treatment in 1.3.4.

The basic idea is to consider the independent variables $t$ and $x$ temporarily as functions of one variable $s$ such that

$$
\frac{d}{d s}=\frac{d t}{d s} \frac{\partial}{\partial t}+\frac{d x}{d s} \frac{\partial}{\partial x}=\frac{\partial}{\partial t}+V(x) \frac{\partial}{\partial x} .
$$

Thus it appears that we have to choose

$$
\begin{aligned}
& \frac{d t}{d s}=1 \Rightarrow t=s+c_{1}, \\
& \frac{d x}{d s}=V(x) \Rightarrow \frac{d x}{V(x)}=d s \Rightarrow G(x)=s+G\left(c_{2}\right) \Rightarrow x=G^{-1}\left(s+G\left(c_{2}\right)\right),
\end{aligned}
$$

where $c_{1}$ and $c_{2}$ are arbitrary constants still at our disposal.

First consider a function $m(t, x)$ satisfying

$$
\frac{\partial m}{\partial t}+V(x) \frac{\partial m}{\partial x}=0 \text {. }
$$

Then $\frac{d \bar{m}}{d s}=0$ where $\bar{m}(s):=m(t(s), x(s))$ and consequently $\bar{m}(s)=$ constant $=\bar{m}(0)$ or

$$
m\left(s+c_{1}, G^{-1}\left(s+G\left(\left(c_{2}\right)\right)\right)=m\left(c_{1}, c_{2}\right) .\right.
$$

The mapping $s \mapsto\left(s+c_{1}, G^{-1}\left(s+G\left(c_{2}\right)\right)\right)$ corresponds to an orbit in the $(t, x)$-plane with starting point $\left(c_{1}, c_{2}\right)$. Such orbits are called characteristics. In our biological model they correspond to the orbits that individual cells follow as a result of their growth.

Next we have to realize that $(t, x)$ points are restricted to the strip $\left\{(t, x) \mid t \geqslant 0, \frac{1}{2} a \leqslant x \leqslant 1\right\}$ and that the homogeneous equation (10.4) only holds in $\left\{(t, x) \mid t \geqslant 0, \frac{1}{2} \leqslant x \leqslant 1\right\}$. The boundary of the latter domain consists of the lines $x=\frac{1}{2}$ and $x=1$ and the segment $t=0, \frac{1}{2} \leqslant x \leqslant 1$. At the line $x=\frac{1}{2}$ and the segment the characteristics enter this domain in the sense that $\left(s+c_{1}, G^{-1}\left(s+G\left(c_{2}\right)\right)\right.$ belongs to the inside for $s>0$ when either $c_{2}=\frac{1}{2}$ or $c_{1}=0$. At the line $x=1$ they leave the domain.

So we can use (10.5) to express $m(t, x)$ for $t \geqslant 0$ and $\frac{1}{2} \leqslant x \leqslant 1$ in terms of $m(0, x)=\phi(x)$ (the initial condition) and $m\left(t, \frac{1}{2}\right)$, which we, pretending that it is a known function, baptize $y(t)$. The procedure is as follows:

i) Take $c_{1}=0$ and $c_{2} \geqslant \frac{1}{2}$ then

$$
m\left(s, G^{-1}\left(s+G\left(c_{2}\right)\right)=\phi\left(c_{2}\right) .\right.
$$

In order to transform back from the variables $\left(s, c_{2}\right)$ to the variables $(t, x)$ we put $(t, x)=\left(s, G^{-1}\left(s+G\left(c_{2}\right)\right)\right)$ and obtain $s=t, c_{2}=G^{-1}(G(x)-t)$ and finally

$$
m(t, x)=\phi\left(G^{-1}(G(x)-t)\right), t \leqslant G(x)-G\left(\frac{1}{2}\right) .
$$


ii) Take $c_{2}=\frac{1}{2}$ and $c_{1}>0$ then

$$
\begin{aligned}
& m\left(s+c_{1}, G^{-1}\left(s+G\left(\frac{1}{2}\right)\right)\right)=y\left(c_{1}\right) . \\
& \text { If }(t, x)=\left(s+c_{1}, G^{-1}\left(s+G\left(\frac{1}{2}\right)\right)\right) \text { then } s=G(x)-G\left(\frac{1}{2}\right) \text { and } c_{1}=t-G(x)+G\left(\frac{1}{2}\right) \text { so that } \\
& m(t, x)=y\left(t-G(x)+G\left(\frac{1}{2}\right)\right), t>G(x)-G\left(\frac{1}{2}\right)
\end{aligned}
$$

This ends our calculations for $\frac{1}{2}<x \leqslant 1$.

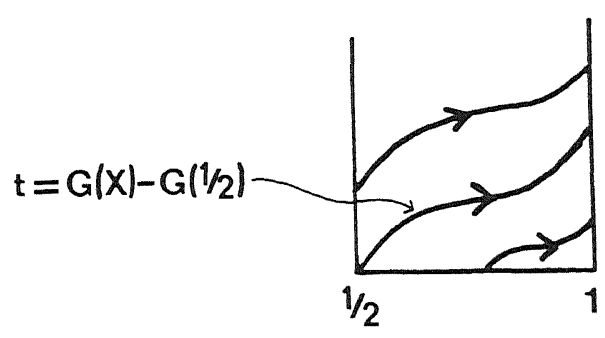

For $\frac{1}{2} a \leqslant x \leqslant \frac{1}{2}$ we have to deal with the inhomogeneous equation

$$
\frac{\partial m}{\partial t}+V(x) \frac{\partial m}{\partial x}=h(t, x)
$$

and the boundary condition

$$
m\left(t, \frac{1}{2} a\right)=0 \text {. }
$$

For the time being $h$ is considered as a known function but later we will substitute $h(t, x)=k(x) m(t, 2 x)$. The characteristics enter the strip $\left\{(t, x) \mid t \geqslant 0, \frac{1}{2} a \leqslant x \leqslant \frac{1}{2}\right\}$ at the segment $t=0, \frac{1}{2} a \leqslant x \leqslant \frac{1}{2}$ and at the line $x=\frac{1}{2} a$, so again we have to distinguish between two choices of coordinates.

i) Take $t=s$ and $x=G^{-1}\left(s+G\left(c_{2}\right)\right)$ with $\frac{1}{2} a \leqslant c_{2} \leqslant \frac{1}{2}$ then

$$
\begin{aligned}
& \left\{\begin{array}{l}
\frac{d \bar{m}}{d s}=h\left(s, G^{-1}\left(s+G\left(c_{2}\right)\right)\right. \\
\bar{m}(0)=m\left(0, c_{2}\right)=\phi\left(c_{2}\right)
\end{array}\right. \\
& \Rightarrow \bar{m}(s)=\phi\left(c_{2}\right)+\int_{0}^{s} h\left(\sigma, G^{-1}\left(\sigma+G\left(c_{2}\right)\right)\right) d \sigma \Rightarrow \\
& m(t, x)=\phi\left(G^{-1}(G(x)-t)\right)+\int_{0}^{t} h\left(\sigma, G^{-1}(\sigma+G(x)-t)\right) d \sigma, \text { for } t \leqslant G(x) .
\end{aligned}
$$

ii) Take $t=s+c_{1}$ and $x=G^{-1}(s)$ with $c_{1} \geqslant 0$ then

$$
\begin{aligned}
& \left\{\begin{array}{l}
\frac{d \bar{m}}{d s}=h\left(s+c_{1}, G^{-1}(s)\right) \\
\bar{m}(0)=m\left(c_{1}, \frac{1}{2} a\right)=0
\end{array}\right. \\
& \Rightarrow \bar{m}(s)=\int_{0}^{s} h\left(\sigma+c_{1}, G^{-1}(\sigma)\right) d \sigma \Rightarrow \\
& m(t, x)=\int_{0}^{G(x)} h\left(\sigma+t-G(x), G^{-1}(\sigma)\right) d \sigma=\int_{a / 2}^{x} h(G(\tau)+t-G(x), \tau) \frac{d \tau}{g(\tau)}, \text { for } t>G(x) .
\end{aligned}
$$

To round off our calculations we have to substitute $h(t, x)=k(x) m(t, 2 x)$, with $m$ given by (10.7) or (10.8), into (10.12) and (10.13), thus expressing $m$ for all $t \geqslant 0, \frac{1}{2} a \leqslant x \leqslant 1$ in terms of the (known) initial condition $\phi$ and the (unknown) function $y$. If our approach is to be consistent, taking $x=\frac{1}{2}$ in (10.12) and (10.13) should produce $y(t)$ ! 
Thus we find an equation for $y$ which, assuming $2 V(x) \geqslant V(2 x)$, takes for $t \geqslant G(1)-G\left(\frac{1}{2}\right)$ the form

$$
y(t)=\int_{a / 2}^{\frac{1}{2}} \frac{k(\tau)}{V(\tau)} y(t+G(\tau)-G(2 \tau)) d \tau .
$$

EXERCISE 10.1. Assume that $2 V(x)>2 V(x)$. Show that

$$
\begin{gathered}
y(t)=\phi\left(G^{-1}\left(G\left(\frac{1}{2}\right)-t\right)\right)+\int_{0}^{t} k\left(G^{-1}\left(\sigma+G\left(\frac{1}{2}\right)-t\right)\right) \phi\left(G^{-1}\left(G\left(2 G^{-1}\left(\sigma+G\left(\frac{1}{2}\right)-t\right)\right)-\sigma\right)\right) d \sigma, t \leqslant G\left(\frac{1}{2}\right), \\
y(t)=\int_{a / 2}^{\frac{1}{2}} \frac{k(\tau)}{V(\tau)} y(t+G(\tau)-G(2 \tau)) d \tau+\int_{\phi(t)}^{\frac{1}{2}} \frac{k(\tau)}{V(\tau)} \phi\left(G^{-1}\left(G(2 \tau)-G(\tau)-t+G\left(\frac{1}{2}\right)\right)\right) d \tau
\end{gathered}
$$

for $G\left(\frac{1}{2}\right) \leqslant t \leqslant G(1)-G\left(\frac{1}{2}\right)$, where $\rho(t)$ is the unique solution of $G(2 \rho)-G(\rho)=t$.

If $2 V(x)>V(2 x)$ we can use the transformation $\sigma=G(2 \tau)-G(\tau)$ and its inverse $\tau=\rho(\sigma)$ to rewrite (10.14) as the Volterra convolution equation

$$
y(t)=\int_{G(a)}^{G(1)-G\left(\frac{1}{2}\right)} \frac{k(\rho(\sigma))}{V(\rho(\sigma))} \frac{d \rho}{d \sigma}(\sigma) y(t-\sigma) d \sigma .
$$

If, on the other hand, $2 V(x)=V(2 x)$, then equation (10.14) is a difference equation (cf. Exercise 9.11)

$$
y(t)=\int_{a / 2}^{\frac{1}{2}} \frac{k(\tau)}{V(\tau)} d \tau y(t-G(a)) .
$$

EXERCISE 10.2. Consider Case II: $V(2 x)=2 V(x)$. Show that

$$
G\left(2 G^{-1}(y)\right)=y+G(a),
$$

and use this result to derive the following relations from (10.7), (10.9), (10.12) and (10.13)

$$
\begin{gathered}
m(t, x)=\left\{\begin{array}{ll}
\phi\left(G^{-1}(G(x)-t)\right) & , t \leqslant G(x)-G\left(\frac{1}{2}\right) \\
y\left(t-G(x)+G\left(\frac{1}{2}\right)\right) & , t>G(x)-G\left(\frac{1}{2}\right)
\end{array}\right\} \quad x>\frac{1}{2} \\
m(t, x)=\left\{\begin{array}{l}
\phi\left(G^{-1}(G(x)-t)\right)+\int_{G^{-1}(G(x)-t)}^{x} \frac{k(\sigma)}{V(\sigma)} d \sigma \phi\left(G^{-1}(G(x)+G(a)-t)\right), t \leqslant G(x) . \\
\int_{a / 2}^{x} \frac{k(\sigma)}{V(\sigma)} d \sigma \phi\left(G^{-1}(G(a)+G(x)-t)\right), G(x)<t \leqslant G(x)+G(a)-G\left(\frac{1}{2}\right) . \\
\int_{a / 2}^{x} \frac{k(\sigma)}{V(\sigma)} d \sigma y\left(t-G(a)+G\left(\frac{1}{2}\right)-G(x)\right), t>G(x)+G(a)-G\left(\frac{1}{2}\right) .
\end{array}\right\} \text { for } x \leqslant \frac{1}{2} .
\end{gathered}
$$

EXERCISE 10.3. Use the relations above to deduce that

$$
\begin{aligned}
& y(t)=\phi\left(G^{-1}\left(G\left(\frac{1}{2}\right)-t\right)\right)+\int_{G^{-1}\left(G\left(\frac{1}{2}\right)-t\right)}^{\frac{1}{2}} \frac{k(\sigma)}{V(\sigma)} d \sigma \phi\left(G^{-1}\left(G\left(\frac{1}{2}\right)+G(a)-t\right)\right), \quad t \leqslant G\left(\frac{1}{2}\right) \\
& y(t)=\int_{a / 2}^{\frac{1}{2}} \frac{k(\sigma)}{V(\sigma)} d \sigma \phi\left(G^{-1}\left(G(a)+G\left(\frac{1}{2}\right)-t\right)\right), \quad G\left(\frac{1}{2}\right)<t \leqslant G(a) .
\end{aligned}
$$


EXERCISE 10.4. Use formulas $(10.20)-(10.23)$ to show that

$$
m(G(a), x)= \begin{cases}\int_{a / 2}^{x} \frac{k(\sigma)}{V(\sigma)} d \sigma\left\{\phi(x)+\int_{x}^{\frac{1}{2}} \frac{k(\sigma)}{V(\sigma)} d \sigma \phi\left(G^{-1}(G(x)+G(a))\right)\right. & , \frac{1}{2} a \leqslant x \leqslant \frac{1}{2}, \\ \int_{a / 2}^{\frac{1}{2}} \frac{k(\sigma)}{V(\sigma)} d \sigma \phi(x) & , \frac{1}{2} \leqslant x \leqslant a, \\ \phi\left(G^{-1}(G(x)-G(a))+\int_{G^{-1}(G(x)-G(a))}^{\frac{1}{2}} \frac{k(\sigma)}{V(\sigma)} d \sigma \phi(x)\right. & , a \leqslant x \leqslant 1 .\end{cases}
$$

REMARKS 10.5. (i) Again we found that relevant features depend crucially on the function $2 V(x)-V(2 x)$.

(ii) The results of this section constitute a first step towards an alternative proof of the existence and uniqueness of solutions. Indeed, one can use (10.15) - (10.17) to give a straightforward constructive proof of the existence of a unique solution $y$ and subsequently $(10.7),(10.9),(10.12)$ and $(10.13)$ to define $m(t, x)$ for $x \neq \frac{1}{2}$. Moreover, the existence of a stable size distribution in case $2 V(x)>V(2 x)$ can be deduced from (10.17) as well (we refer to Chapter IV section 2 for an exposition of the relevant material). In Case II $(V(2 x)=2 V(x))$ formulas $(10.18)-(10.23)$ in fact provide us with an explicit representation of the solution.

(iii) We were able to derive a scalar equation for $y(t)=m\left(t, \frac{1}{2}\right)$ since every potential mother cell necessarily passes size $\frac{1}{2}$ during her life time (recall the reflections about the interpretation of $\pi(0)$ in Interlude 4.3.2 in Chapter I. Here the Assumption 4.2: $a \geqslant \frac{1}{2}$ is essential. If we relax this assumption to $a \geqslant 2^{-k}$ we need $k$ equations for the variables $m\left(t, 2^{-l}\right), l=1, \ldots, k$.

EXERCISE 10.6. Assume $a \geqslant \frac{1}{4}$ and $2 V(x) \geqslant V(2 x)$. Derive the analogue of equation (10.14).

\section{The merry-go-round}

In this section we concentrate on Case II of Definition 5.2.: $V(2 x)=2 V(x)$. Firstly, we summarize the knowledge obtained so far. The characteristic equation has countably many simple roots

$$
\lambda_{l}=\delta+\frac{2 l \pi i}{\tau}, l \in \mathbb{Z},
$$

where

$$
\delta=\frac{1}{\tau} \ln \int_{a / 2}^{1 / 2} \frac{k(\xi)}{V(\xi)} d \xi .
$$

and by definition

$$
\tau=G(a)
$$

the size doubling time (see Exercise 9.11). The corresponding eigenvectors are

$$
\psi_{l}(x)=\theta(x) e^{\lambda_{l}\left(G\left(\frac{1}{2}\right)-G(x)\right)}
$$

where

$$
\theta(x)=\frac{\int_{a / 2}^{x} \frac{k(\xi)}{V(\xi)} d \xi}{\int_{a / 2}^{\frac{1}{2}} \frac{k(\xi)}{V(\xi)} d \xi}
$$

(with the usual convention that $k(x)=0$ for $x>\frac{1}{2}$ ).

From (10.20) and (10.21) it follows that for $t>\tau$

$$
m(t, x)=\theta(x) y\left(t-G(x)+G\left(\frac{1}{2}\right)\right)
$$


where $y$ is a solution of

$$
y(t)=\int_{a / 2}^{\frac{1}{2}} \frac{k(\sigma)}{V(\sigma)} d \sigma y(t-\tau), t>\tau,
$$

(with $y(t)$ for $0 \leqslant t \leqslant \tau$ determined by the initial function $\phi$ as described in (10.22), (10.23)).

Thus an obvious conjecture is that the action of the semigroup is some kind of combination of multiplication and periodic continuation, and our objective is to verify this conjecture while simultaneously making it more precise. In order to separate the multiplication from the periodic continuation we introduce

$$
z(t)=e^{-\delta t} y(t)
$$

and find upon substitution into (11.6) that

$$
z(t)=z(t-\tau), t \geqslant \tau,
$$

which implies that $z$ is a $\tau$ periodic function. Hence

$$
m(t, x)=e^{\delta t} \theta(x) e^{\delta\left(G\left(\frac{1}{2}\right)-G(x)\right)} z\left(t-G(x)+G\left(\frac{1}{2}\right)\right)
$$

or, in words: for $t \geqslant \tau, T(t) \phi$ is the product of three factors

i) $e^{\delta t}$ : an exponential function of time with exponent $\delta$

ii) $\theta(x) e^{\delta\left(G\left(\frac{1}{2}\right)-G(x)\right)}$ : a fixed element of $X$

iii) $z\left(t-G(x)+G\left(\frac{1}{2}\right)\right):$ a $\tau$-periodic function, with argument $t-G(x)+G\left(\frac{1}{2}\right)$, which is the only factor that depends on the specific initial condition $\phi$.

Note that (11.9) shows that $\omega_{0}=\delta=s(A)$ and in addition (since $|\kappa L|_{\alpha}=\kappa|L|_{\alpha}$ for $\kappa>0$ ) that $\omega_{e}=\delta$ as well. An alternative proof of the latter identity is given by the observation that $\left\{e^{\lambda_{t} t} \mid l \in \mathbb{Z}\right\}$ lies dense on the circumference $\left\{\lambda|| \lambda \mid=e^{\delta t}\right\}$ when $t / \tau$ is irrational (indeed, use the definition of essential spectrum, Theorem 8.11 (ii) and $\omega_{e} \leqslant \omega_{0}=\delta$ ).

Recalling Theorem 7.8 we observe that $\Im\left(e^{\delta \tau} I-T(\tau)\right)$ is the infinite dimensional subspace which is obtained by taking the closure of the set spanned by

$$
\psi_{l}(x)=\theta(x) e^{\delta\left(G\left(\frac{1}{2}\right)-G(x)\right)} e^{\frac{2 l \pi i}{\tau}\left(G\left(\frac{1}{2}\right)-G(x)\right)}, l \in \mathbb{Z} .
$$

Well known results from Fourier analysis imply that this is precisely the subspace of functions of the form

$$
\theta(x) e^{-\delta G(x)} q(G(x)),
$$

with $q$ a continuous $\tau$-periodic function. Clearly (11.9) implies that $T(t) \phi$ belongs to this subspace for $t \geqslant \tau$.

It follows likewise from Fourier theory that the sequence $\psi_{l}$ does not constitute a basis for $\mathscr{T}\left(e^{\delta \tau} I-T(\tau)\right)$, i.e. the expansion in a series $\sum c_{l} \psi_{l}$ does not necessarily converge in our supremum norm topology!

Apart from some transient phenomena during a time interval of length $\tau$, the dynamics takes place in the dominant subspace $\vartheta\left(e^{\delta \tau} I-T(\tau)\right)$. Since this is an infinite dimensional subspace, infinitely many characteristics of the initial condition (like zeros, extrema etc.) remain manifest for all time. This is in sharp contrast with the onedimensional asymptotic dynamics in the case $V(2 x)<2 V(x)$.

A priori the existence of a canonical (i.e. commuting with the semigroup) projection $P$ onto $\tau\left(e^{\delta \tau} I-T(\tau)\right)$ is not guaranteed. Calculations involving the residues in the isolated poles $\lambda_{l}$ are not directly applicable since the resulting series might be divergent. (A remedy for this deficiency is provided by Cesaro summation (this was pointed out to the author by Prof. R. Nagel; see Schaffer, 19 1974, III.7).) Happily, however, we can in the present case easily obtain an explicit representation of $P$ from a detour: given $\phi$ we can calculate $T(\tau) \phi$ from $(10.24)$ and subsequently determine $P \phi \in \mathscr{U}\left(e^{\delta \tau} I-T(\tau)\right)$ from the condition that $T(\tau) P \phi=T(\tau) \phi$, by exploiting the fact that (11.9) gives an explicit expression for the (group) action of $T(t)$ on $\Re\left(e^{\delta \tau} I-T(\tau)\right)$. Thus we obtain*

- Side-remark: For readers interested in functional differential equations we point out that for these the corresponding construction cannot be carried out because one has neither the precise characterization of the relevant subspace nor the explicit representation of the dynamics on it. See VERDUYN-LUNEL (1984) for a recent treatment of retarded functional differential equations admitting solutions that vanish after finite time. 


$$
(P \phi)(x)= \begin{cases}\theta(x)\left\{\phi(x)+\int_{x}^{\frac{1}{2}} \frac{k(\sigma)}{V(\sigma)} d \sigma \cdot \phi\left(G^{-1}(G(x)+G(a))\right)\right\} & , \frac{1}{2} a \leqslant x \leqslant \frac{1}{2}, \\ \phi(x) & , \frac{1}{2} \leqslant x \leqslant a, \\ \frac{1}{\int_{a / 2}^{\frac{1}{2}} \frac{k(\sigma)}{V(\sigma)} d \sigma}\left\{\phi\left(G^{-1}(G(x)-G(a))\right)+\int_{G^{-1}(G(x)-G(a))}^{\frac{1}{2}} \frac{k(\sigma)}{V(\sigma)} d \sigma \cdot \phi(x)\right\} & , a \leqslant x \leqslant 1 .\end{cases}
$$

EXERCISE 11.1. Verify that $P^{2}=P$, that $\mathscr{R}(P) \subset \mathscr{T}\left(e^{\delta \tau} I-T(\tau)\right)$ and that the restriction of $P$ to $\mathscr{R}\left(e^{\delta \tau} I-T(\tau)\right)$ reduces to the identity.

REMARK 11.2. Put a tape in a loop and slowly but surely turn the loudspeakers on. What you hear resembles to some extent the meaning of (11.9). Another convenient mental picture is the spiral staircase. Recall from Exercise 9.11 that the phase period $\tau=G(a)$ equals the time which individual cells need to double their size.

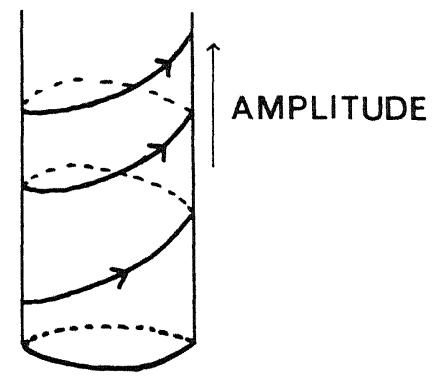

EXERCISE 11.3. In Exercise I.4.3.6 the equation

$$
\frac{\partial}{\partial t} n(t, x)=-\frac{\partial}{\partial x}(V(x) n(t, x))-\mu(x) n(t, x)-b(x) n(t, x)+2 \int_{x}^{1} \frac{d(p)}{p} b\left(\frac{x}{p}\right) n\left(t, \frac{x}{p}\right) d p
$$

was derived as a description of cell proliferation, when the probability that a mother cell of size $x$ splits into one daughter of size $p x$ and one of size $(1-p) x$ is given by the $x$-independent probability density $d(p)$. Assume that $d(p)=0$ for $p \notin\left(\frac{1}{2}-\Delta, \frac{1}{2}+\Delta\right)$ for some $\Delta \in\left(0, \frac{1}{2}\right)$ and that $a>\frac{1}{2}+\Delta$, where, as before, $b(x)=0$ for $x \leqslant a$ and $b(x)>0$ for $x>a$. Derive the characteristic equation and analyse it. Put special emphasis on the case $V(x)=x$. Cautionary note: the minimal size is now $\left(\frac{1}{2}-\Delta\right) a$.

EXERCISE 11.4. In the special case that fission occurs exactly when reaching size $x=1$ the analogue of (11.12) takes the form

$$
\frac{\partial n}{\partial t}(t, x)=-\frac{\partial}{\partial x}(V(x) n(t, x))-\mu(x) n(t, x)+2 d(x) V(1) n(t, 1),
$$

supplemented by the boundary condition

$$
n\left(t, \frac{1}{2}-\Delta\right)=0
$$

Analyse this problem.

Remark 11.5. An alternative way to derive (11.9) goes as follows. Put

$$
m(t, x)=e^{\delta t} \theta(x) e^{\delta\left(G\left(\frac{1}{2}\right)-G(x)\right)} p(t, x)
$$

then

$$
\frac{\partial p}{\partial t}(t, x)+V(x) \frac{\partial p}{\partial x}(t, x)=\frac{k(x) e^{-\delta \tau}}{\theta(x)}[p(t, 2 x)-p(t, x)]
$$


Since $\theta\left(\frac{1}{2} a\right)=0$ we are led to require $p\left(t, \frac{1}{2} a\right)=p(t, a)$ in order to keep the right hand side bounded. If $p(t, 2 x)=p(t, x)$ the right hand side reduces to zero and therefore $p(t, x)=p\left(t-G(x), \frac{1}{2} a\right)=p(t-G(x), a)$. Hence $p(t, a)=p(t-\tau, a)$, i.e. $p(t, a)$ is a $\tau$-periodic function. Consistency now requires that $p(t, 2 x)=p(t-G(2 x), a)=p(t-G(x), a),=p(t, x)$ but this requirement is fulfilled since $G(2 x)-G(x)=G(a)$. Thus we can solve (11.16) explicitly after a transient period of length $\tau=G(a)$.

\section{The merry-go-round with an absorbing exit}

In Case III of Definition $5.2 V$ satisfies the relation $V(2 x)=2 V(x)$ on the interval $\frac{1}{2} a \leqslant x \leqslant \beta$ while $V(2 x)<2 V(x)$ for $\beta<x \leqslant \frac{1}{2}$. Here $\beta$ is some number between $\frac{1}{2} a$ and $\frac{1}{2}$. Because of Theorem 5.8 (the existence of a strictly dominant real eigenvalue $\lambda_{d}$ ) we expect that in this case the normalized size distribution will converge towards a stable distribution. But calculations as in Exercise 9.8 indicate that $T(t)$ will not be compact after finite time. Therefore we need more subtle arguments involving the measure of non-compactness and in particular the result $\omega_{0}=\max \left\{\omega_{e}, \omega_{n}\right\}$ (Theorem 8.12). Remarkably our mathematical procedure can to a large extent be described in biological terms and this we will do first.

One can conceive of the population as the union of two subpopulations. The first of these consists of those cells which were either present at $t=0$ or arose from divisions of ancestors which at the moment of division had a size smaller than or equal to $2 \beta$ (in other words, none of the ancestors has divided after $t=0$ with size greater than $2 \beta$ ). The second subpopulation is the complement and consists of those cells for which at least one of the ancestors has undergone a division after $t=0$, while having a size greater than $2 \beta$.

The dynamics of the first subpopulation is of the merry-go-round type. The members of the second subpopulation, on the other hand, are obtained by successive application of generation operators of which one at least is compact, and hence the product is compact. Moreover, the traffic between the two subpopulations goes one way only since offspring of the first subpopulation can be a member of the second but not vice-versa. Therefore the second will grow faster than the first (indeed, the chance that an arbitrary newborn cell that is determined to divide will do so with size less than or equal to $2 \beta$ is less than one and $q^{\prime} \rightarrow 0$ when $l \rightarrow \infty$ and $|q|<1$ !). Hence there is hope that the compact part of the semigroup operator is asymptotically dominant over the non-compact part in the sense that $\omega_{e}<\omega_{0}$.

Notation: we shall denote functions that describe the first subpopulation by a dash above the letter, e.g. $\bar{m}$, and functions that describe the second subpopulation by a hat, e.g. $\hat{m}$.

Define

$$
\bar{k}(x)=\left\{\begin{array}{cc}
k(x) & , \frac{1}{2} a \leqslant x \leqslant \beta, \\
0 & , \beta<x \leqslant 1,
\end{array}\right.
$$

and

$$
\hat{k}(x)= \begin{cases}k(x) & , \beta<x \leqslant \frac{1}{2}, \\ 0 & , \frac{1}{2}<x \leqslant 1 .\end{cases}
$$

Let $m$ satisfy

$$
\left\{\begin{array}{l}
\frac{\partial m}{\partial t}+V(x) \frac{\partial m}{\partial x}=k(x) m(t, 2 x) \\
m(0, x)=\phi(x)
\end{array}\right.
$$

then we may write $m=\bar{m}+\hat{m}$ where

$$
\begin{aligned}
& \left\{\begin{array}{l}
\frac{\partial \bar{m}}{\partial t}+V(x) \frac{\partial \bar{m}}{\partial x}=\bar{k}(x) \bar{m}(t, 2 x) \\
\bar{m}(0, x)=\phi(x)
\end{array}\right. \\
& \left\{\begin{array}{l}
\frac{\partial \hat{m}}{\partial t}+V(x) \frac{\partial \hat{m}}{\partial x}=k(x) \hat{m}(t, 2 x)+\hat{k}(x) \bar{m}(t, 2 x) \\
\hat{m}(0, x)=0
\end{array}\right.
\end{aligned}
$$


The definition

$$
\bar{T}(t) \phi=\bar{m}(t, \cdot ; \phi)
$$

produces a merry-go-round semigroup and consequently

$$
\omega_{0}(\bar{T}(t))=\frac{1}{G(a)} \ln \int_{a / 2}^{1 / 2} \frac{\bar{k}(\xi)}{V(\xi)} d \xi=\frac{1}{G(a)} \ln \int_{a / 2}^{\beta} \frac{k(\xi)}{V(\xi)} d \xi .
$$

Next we define

$$
\hat{U}_{1}(t) \phi=\int_{0}^{t} U_{0}(t-\tau) \hat{C} U_{0}(\tau) \phi d \tau
$$

where

$$
(\hat{C} \phi)(x)=\hat{k}(x) \phi(2 x) .
$$

Explicitly we have (cf. Exercise 9.1)

$$
\left(\hat{U}_{1}(t) \phi\right)(x)=\int_{0}^{t} \hat{k}(X(-t+\tau, x)) \phi(X(-\tau, 2 X(-t+\tau, x))) d \tau .
$$

Now the point is that we can perform the transformation of Exercise 9.2 for precisely the $\tau$-domain for which $\hat{k}(X(-t+\tau, x)) \neq 0$ and consequently the compactness of $\hat{U}_{1}(t)$ follows in precisely the same way as the compactness of the operator $U_{1}(t)$ of section 9 . Let

$$
\hat{T}(t) \phi=\hat{m}(t, ; \phi)
$$

(nota bene that $\{\hat{T}(t)\}$ is not a semigroup) then repetition of the argument yields that $\hat{T}(t)$ is compact for all $t$. From

$$
T(t)=\bar{T}(t)+\hat{T}(t)
$$

we infer that $|T(t)|_{\alpha}=|\bar{T}(t)|_{\alpha}$ and so

$$
\omega_{e}(T(t))=\lim _{t \rightarrow \infty} \frac{\log |T(t)|_{\alpha}}{t}=\lim _{t \rightarrow \infty} \frac{\log |\bar{T}(t)|_{\alpha}}{t} \leqslant \omega_{0}(\bar{T}(t)) .
$$

EXERCISE 12.1. (i) Use the definition (4.4) of $\pi(\lambda)$ and the definition (12.1) of $\bar{k}$ to show that $\lambda_{d}(k)>\lambda_{d}(\bar{k})$. (ii) Deduce from (i) that (recall the definition $s(A)=\sup \{\operatorname{Re} \lambda \mid \lambda \in \sigma(A)\}) s(A)>s(\bar{A})$. (iii) Use $s(\bar{A})=\omega_{0}(\bar{T}(t)$ ) and (ii) to obtain $s(A)>\omega_{e}(\bar{T}(t))=\omega_{e}(T(t))$ and conclude that $\omega_{0}(T(t))=s(A)=\lambda_{d}(k)$ and, moreover, $\omega_{e}(T(t)) \leqslant \lambda_{d}(k)-\epsilon$ for some $\epsilon>0$.

Let as before $T_{R}(t)$ denote the restriction of $T(t)$ to the invariant subspace $\operatorname{R}\left(\lambda_{d} I-A\right)$ then $\omega_{0}\left(T_{R}(t)\right)=\max \left\{\omega_{e}\left(T_{R}(t)\right), s\left(A_{R}\right)\right\} \leqslant \max \left\{\omega_{e}(T(t)), s\left(A_{R}\right)\right\} \leqslant \lambda_{d}-\epsilon$ for some $\epsilon>0$. It follows that $T_{R}(t)$ satisfies an exponential estimate with exponent $\lambda_{d}-\epsilon$, where $\epsilon$ is the minimum of the distances along the real axis to, on the one hand, the other eigenvalues and, on the other hand, the essential spectrum. We summarize the result in

THEOREM 12.2. With $V$ as in Case III of Definition 5.2 the conclusion of Theorem 9.12 about the stable size distribution remains valid, but the characterization of the exponent in the remainder term has to be modified as indicated above.

The assumption on $V$ in Case III is such that the set $\left\{x \mid V(2 x)=2 V(x), \frac{1}{2} a \leqslant x \leqslant \frac{1}{2}\right\}$ is just one interval and, moreover, such that the complement in $\left[\frac{1}{2} a, \frac{1}{2}\right]$ is just one interval as well. In the general case in which $V(2 x) \neq 2 V(x)$ for some $x \in\left[\frac{1}{2} a, \frac{1}{2}\right]$, these sets might consist of many intervals and in addition $V(2 x)-2 V(x)$ might assume both positive and negative values. This complicates the notation and the presentation of the arguments, but apart from this one can use essentially the ideas of this section to prove

THEOREM 12.3. A necessary and sufficient condition for the existence of a stable size distribution is that $V(2 x) \neq 2 V(x)$ for at least one $x \in\left[\frac{1}{2} a, 1\right]$.

A somewhat different proof of this result is presented in Diekmann, HeIJMans \& ThIEME (1984, part II), a paper which mainly deals with extensions of the above results to the case of time-periodic rates $b, \mu$ and $V$. 


\section{Remarks about positivity}

Performing explicit calculations we derived the characteristic equation $\pi(\lambda)=1$ for the spectrum of the generator $A$ and subsequently we found from an analysis of $\pi$ that two possibilities exist:

(i) either there exists a real eigenvalue $\lambda_{d}$ which is strictly dominant in the sense that $\operatorname{Re} \lambda \leqslant \lambda_{d}-\epsilon$ for all spectral values $\lambda \neq \lambda_{d}$ and some $\epsilon>0$, or

(ii) there exists a vertical line $\operatorname{Re} \lambda=\delta$ on which lie countably many eigenvalues which constitute an additive subgroup of $\mathbb{R}$ in the sense that $\delta+i l \eta, l \in \mathbb{Z}$, is an eigenvalue whenever $\delta+i \eta$ is; and all other eigenvalues (if any; there were none in our case) satisfy $\operatorname{Re} \lambda \leqslant \delta-\epsilon$ for some $\epsilon>0$.

The aim of this section is to draw attention to the fact that it is frequently possible, notably in population problems, to obtain such conclusions even when explicit calculations are impossible or just cumbersome. The mathematical theory which deals with such matters goes under the heading of "spectral theory of positive operators and positive semigroups". Note that clearly $T(t)$ maps positive functions onto positive functions as required by our interpretation of $T(t) \phi$ as a population density. The set of all positive functions in $X$ is an example of a cone and an operator is called positive if it maps some cone into itself. As a generalization of the famous Perron-Frobenius theorem on the eigenvalues of a matrix with positive entries, there exists a collection of results which describes to some extent the structure of the spectrum of a positive operator (SCHAEFER, 1974). Moreover, analogues of such results for positive semigroups and their generators are known (GREINER, 1981; also see NAGEL, 1984, GrEINER, 1984, and the references given there). The fact that we did not need these results in the present chapter (simply because we had other means to analyse the spectrum) detracts nothing from the merits of positive operator theory in the context of structured population models. Indeed, in Chapter $\mathrm{V}$ positivity arguments will play a major role and we refer to that chapter for an outline of the relevant theory. In addition we refer to the paper "Structured populations, linear semigroups and positivity" (Heumans, 1984a) for a systematic exposition and a wealth of examples.

\section{A somewhat special nonlinear problem}

In this section we show how the conclusions about the model for substrate limited growth in the chemostat presented in subsection I.4.5 can be derived from the linear theory developed so far. At the risk of causing confusion we shall now use again the symbols $A$ and $T(t)$ in the context of the original variables (recall the transformation (1.6)).

Abstractly we can write the balance equation (I.4.5.5) in the form (with $S$ instead of $R$ for the substrate concentration)

$$
\frac{d n}{d t}=\beta(S) A n-D n
$$

where

$$
(A \phi)(x)=-(V(x) \phi(x))^{\prime}-V(x) \delta(x) \phi(x)+4 V(2 x) \delta(2 x) \phi(2 x)
$$

with the appropriate domain of definition (so note that this $A$ corresponds to the untransformed problem and is different from, but intertwined with, the $A$ of the foregoing sections). We know that $A$ generates a linear semigroup, let us call it $T(t)$, on a space of continuous functions with a tailor-made norm (1.11). Pretending that the substrate concentration $S$ is a known function of time we can solve the equation for $n$ quasi-explicitly:

$$
n(t, \cdot ; \phi)=e^{-D t} T\left(\int_{0}^{t} \beta(S(\tau)) d \tau\right) \phi,
$$

where $\phi$ denotes the initial condition for $n$. As a side remark we mention that (14.3) delivers us from the obligation to define the notion of "a solution" for the nonlinear problem: we simply refer back to the end of section 3 .

Since $\beta(S(t)) \geqslant 0$ the integral $\int_{0}^{t} \beta(S(\tau)) d \tau$ approaches a limit as $t \rightarrow \infty$. If this limit would be finite then necessarily we would have that $S(t) \rightarrow 0$ for $t \rightarrow \infty$. But then (14.3) implies that the biomass $W(t) \rightarrow 0$ as well and hence $Z(t)=\alpha W(t)+S(t) \rightarrow 0$ which is in contradiction with the result of Exercise 1.4.5.3. We conclude that $\int_{0}^{t} \beta(S(\tau)) d \tau \rightarrow \infty$ for $t \rightarrow \infty$. Assuming that for some $x \in\left[\frac{1}{2} a, \frac{1}{2}\right], V(2 x) \neq 2 V(x)$, this implies that 


$$
n(t, \cdot ; \phi)=\rho(t)\left\{\phi_{d}+o(1)\right\}, t \rightarrow \infty,
$$

where $\phi_{d}$ is the stable size distribution and $\rho(t)$ a real valued function which needs further investigation. Note that for constant death rates $\mu$ the stable distribution does not depend on the precise value of the death rate although, of course, the dominant eigenvalue does; if below we write $\lambda_{d}$ we mean the dominant eigenvalue corresponding to $\mu=0$.

Substituting (14.4) into the differential equation for $n$ we find

$$
\frac{d \rho}{d t}=\left(\lambda_{d} \beta(S)-D\right) \rho
$$

EXERCISE 14.1. Verify that the $o(1)$ term is rightly left out of (14.5) since the o(1) term in (14.4) lies in $\Re\left(\lambda_{d} I-A\right)$ while $\mathscr{R}\left(\lambda_{d} I-A\right) \cap \Re\left(\lambda_{d} I-A\right)=\{0\}$.

Substitution into the differential equation (I.4.5.6) for $S$ leads to

$$
\frac{d S}{d t}=D\left(S^{i}-S\right)-\alpha_{o} \beta(S) \rho+\beta(S) \rho \cdot o(1),
$$

where

$$
\alpha_{0}=\alpha \int_{a / 2}^{1} V(\xi) \phi_{d}(\xi) d \xi .
$$

Because $Z(t)=\alpha W(t)+S(t)$ remains bounded for $t \rightarrow \infty$ and both $W$ and $S$ are positive, each of them remains bounded as well. The boundedness of $W$ implies the boundedness of $\rho$. So as far as the asymptotic behaviour is concerned we may forget about the $\beta(S) \rho o(1)$ term in the equation for $\frac{d S}{d t}$. We now refer back to subsection I.4.5, and in particular to the Exercises 4.5.6 and 4.5.7, for a formulation of the conclusions which can be drawn. 